\title{
An integrative systems biology approach to identify the molecular basis of sperm quality in swine
}

\section{Marta Godia}

Centre for Research in Agricultural Genomics CRAG (CSIC-IRTA-UAB-UB)

\section{Antonio Reverter}

CSIRO Queensland Bioscience Precint Camrody

\section{Rayner Gonzalez-Prendes}

Centre for Research in Agricultural Genomics CRAG (CSIC-IRTA-UAB-UB)

\section{Yuliaxis Ramayo-Caldas}

Institut de Recerca i Tecnologia Agroalimentaries

\section{Anna Castello}

Centre for Research in Agricultural Genomics CRAG (CSIC-IRTA-UAB-UB)

Joan Enric Rodriguez-Gil

Universitat Autonoma de Barcelona

Armand Sanchez

Universitat Autonoma de Barcelona

Alex Clop ( $\square$ alex.clop@cragenomica.es )

CRAG https://orcid.org/0000-0001-9238-2728

\section{Research}

Keywords: sperm quality, systems biology, sperm RNA, GWAS, swine, small RNA-seq, miRNA

Posted Date: March 30th, 2020

DOI: https://doi.org/10.21203/rs.3.rs-19366/v1

License: (c) (1) This work is licensed under a Creative Commons Attribution 4.0 International License.

Read Full License 
1 An integrative systems biology approach to identify the molecular basis of

2 sperm quality in swine

3 Marta Gòdia1, Antonio Reverter2, Rayner González-Prendes1, Yuliaxis Ramayo-

4 Caldas3, Anna Castelló1,4, Joan-Enric Rodríguez-Gil5, Armand Sánchez4 and Alex

5 Clop $1,6, *$

6

7 Affiliations:

8 1Animal Genomics Group, Centre for Research in Agricultural Genomics (CRAG)

9 CSIC-IRTA-UAB-UB, Campus UAB, 08193, Cerdanyola del Vallès (Barcelona),

10 Spain.

112 CSIRO Agriculture and Food, Queensland Bioscience Precinct, 306 Carmody Rd.,

12 St. Lucia, Brisbane, QLD, 4067, Australia.

13 3Animal Breeding and Genetics Program, Institute for Research and Technology in

14 Food and Agriculture (IRTA), Torre Marimon, 08140, Caldes de Montbui, Catalonia,

15 Spain.

164 Unit of Animal Science, Department of Animal and Food Science, Autonomous

17 University of Barcelona, 08193, Cerdanyola del Vallès (Barcelona), Catalonia, Spain

185 Unit of Animal Reproduction, Department of Animal Medicine and Surgery,

19 Autonomous University of Barcelona, 08193, Cerdanyola del Vallès (Barcelona),

20 Catalonia, Spain

216 Consejo Superior de Investigaciones Científicas (CSIC), 08003, Barcelona,

22 Catalonia, Spain.

$23{ }^{*}$ Corresponding author: alex.clop@cragenomica.es 
24 Abstract

25 Background:

26 Genetic pressure in animal breeding is sparking the interest to select for elite boars

27 with higher sperm quality to maximize ejaculate doses and fertility rates. However, 28 the molecular basis of sperm quality remains largely unexplored. In this study, we 29 sought to identify candidate genes, pathways and DNA variants associated to sperm 30 quality in swine by analyzing 25 sperm-related phenotypes using a systems biology 31 approach that integrates GWAS and RNA-seq.

32 Results:

33 By GWAS, we identified 12 QTL regions associated to the percentage of head and 34 neck abnormalities, abnormal acrosomes and motile spermatozoa. Candidate genes included CHD2, KATNAL2, SLC14A2 or ABCA1. By RNA-seq, we detected 6,128 significant correlations between sperm traits and gene RNA abundances. We built a gene interaction network with the GWAS and the RNA-seq data. To build a robust gene interaction network, only the pair-wise interactions present in both the genetic co-association and the RNA co-abundance network were kept. Moreover, we also included to the Final Network both the genes which RNA abundances correlated with more than 4 semen traits as well as the miRNAs interacting with the genes on the network. The Final Network was enriched for genes involved in gamete generation and development, meiotic cell cycle, DNA repair or embryo implantation. We finally designed a panel of 73 SNPs provided from the GWAS, eGWAS and the Final Network, that explains between 5 to $36 \%$ of the phenotypic variance of the sperm 46 traits.

\section{Conclusions:}


48 By means of a systems biology approach, we identified potential key genes affecting

49 sperm quality. Furthermore, we propose a SNP panel that might explain a

50 substantial part of the genetic variance for semen quality in swine and may thus be

51 of interest for the pig breeding sector.

52

53 Keywords: sperm quality, systems biology, sperm RNA, GWAS, swine, small RNA54 seq, miRNA 


\section{Background}

Sperm carries the paternal genome and a wide repertoire of molecules including

RNAs, which are essential for fertilization and the development of a new organism.

Spermatogenesis, the process whereby germ cells proliferate and develop into mature spermatozoa, is controlled by multiple factors. Both DNA polymorphisms and gene expression have been linked to sperm quality and/or fertility in several mammalian species including cattle (Boe-Hansen et al., 2018) and swine (reviewed in: Gòdia et al., 2018a; Krausz et al., 2015). High-quality sperm is decisive to maximize the propagation of the best genetic material in livestock and the sustainability of the pig breeding sector. For this reason, ejaculated sperm is subjected to strict quality filters in boar artificial insemination (Al) studs. Al farms regularly evaluate the quality of ejaculates measuring traits such as concentration, morphology, viability and motility kinetics, as a way to predict their fertilizing ability (Gadea, 2005). Although these traits have been found to be low to moderately heritable (Diniz et al., 2014; Marques et al., 2018; Smital et al., 2005; Wolf, 2009), the molecular processes and genetic mechanisms controlling sperm quality are far from being fully understood and boar replacement due to insufficient sperm quality remains an economic hurdle for the sector (Robinson and Buhr, 2005).

Currently, there are few studies employing high-throughput techniques to investigate the genetic basis of sperm quality in swine. To date, 5 genome-wide association studies (GWAS) have been carried. Diniz et al. (Diniz et al., 2014) identified a single quantitative trait loci $(\mathrm{QTL})$ region associated to sperm motility in Large White. Two years later, Zhao and collaborators (Zhao et al., 2016) reported 3 multi-SNP QTL regions associated with epididymal weight, sperm concentration and total sperm per ejaculate, respectively and 7 singleton QTLs related to sperm motility, semen 
80 temperature, seminiferous tubule diameter and number of ejaculates in a White

81 Duroc x Erhualian F2 population. Marques et al. (Marques et al., 2018) detected 16 and 6 QTL regions in Large White and Landrace, respectively, associated with sperm motility, number of cells per ejaculate and morphological abnormalities. More recently, several QTL regions have been identified in a Duroc population associated to the number of sperm cells, sperm motility, sperm progressive motility, total morphological abnormalities, coiled tail, bent tail, proximal droplets, distal droplets and distal midpiece reflex (Gao et al., 2019; Zhao et al., 2020).

The presence of RNA molecules in the boar sperm is well documented (Gòdia et al., 2018b; Gòdia et al., 2019a), but their relation to sperm quality has been just tinely explored. Porcine sperm RNAs are highly fragmented and their gene abundances are mostly associated to prior transcriptional events linked to spermatogenesis, fertility and embryo development (Gòdia et al., 2019a). A complex suite of RNAs are comprised in sperm, including coding (mRNA), long noncoding RNAs (e.g. circular RNA -circRNA-) and short noncoding RNAs (e.g. microRNA - miRNA- or Piwi interacting RNA -piRNA-) (Gòdia et al., 2019a). Several studies have reported a relation between RNA abundances and semen quality in mammals (Capra et al., 2017; Jodar et al., 2015; Wang et al., 2019). In swine, Curry et al. performed quantitative RT-PCR (RT-qPCR) targeting 10 miRNAs and identified 5 and 2 miRNAs associated to sperm morphology and motility, respectively (Curry et al.,

100 2011). Moreover, our group has also identified a correlation between the abundance 101 of some circRNAs (Gòdia et al., 2019b) and piRNAs (Ablondi et al., 2020) with semen quality parameters in the porcine species.

103 Resulting from this recent work, it is now apparent that the genetic complexity of 104 sperm quality involves several molecular mechanisms and pathways that are highly 
105 interconnected. For this reason, a systems biology approach to assess gene

106 connections and functional interactions using genomics and transcriptomics is an

107 attractive alternative to the classical "one-gene one-trait" analysis of a stand-alone

108 GWAS or a differential gene expression analysis. Evaluating modules of interacting

109 genes rather than single genes can provide a wider and more holistic picture to

110 predict their functions and the regulation of complex traits (Cho et al., 2012).

111 Furthermore, it can be used to design knowledge-based technologies and tools for

112 their application to animal breeding.

113 The aim of this study was to identify candidate genes, pathways and DNA variants

114 associated to sperm quality in pigs integrating in a systems biology approach, GWAS

115 and RNA-seq.

116

117 Methods

118 Sample collection and phenotype measurement

119 Three hundred fresh sperm ejaculates each from a different Pietrain boar from

120 commercial farms were collected by specialized professionals between September

1212014 and January 2017. Sperm was obtained using the hand glove method,

122 immediately diluted (1:2) in commercial extender and kept at $16^{\circ} \mathrm{C}$ for up to 2 hours

123 until phenotype assessment. Blood samples were collected from specialists during

124 their routine sample collection and gDNA was extracted using a phenol-chloroform

125 based method. The ejaculates were purified to remove somatic cells as described

126 previously (Gòdia et al., 2018b) and purified spermatozoa were stored with Trizol® at

$127-80^{\circ} \mathrm{C}$ until further use.

128 Phenotypic records from fresh sperm were measured as previously described (Gòdia

129 et al., 2018b) and included: sperm concentration (CON), the percentage of viable 
130 cells (VIAB), the percentage of morphologically abnormal acrosomes (ACRO),

131 osmotic resistance test (ORT), the percentage of morphologically abnormal sperm

132 cells (of the head $-\mathrm{HABN}$-, neck $-\mathrm{NABN}$ - and tail $-\mathrm{TABN}-$ ) and of cells with

133 cytoplasmatic droplets (proximal -PDROP- and distal -DDROP-). Sperm motility

134 traits were also assessed using the computer-assisted semen analysis (CASA)

135 system (Integrated Sperm Analysis System V1.0; Proiser) and included the

136 percentage of motile spermatozoa cells (MT) (with Average Path Velocity -VAP- > 10

$137 \mu \mathrm{m} / \mathrm{s})$, average Curvilinear Velocity $(\mathrm{VCL})(\mu \mathrm{m} / \mathrm{s})$, average Straight Line Velocity

$138(\mathrm{VSL})(\mu \mathrm{m} / \mathrm{s})$ and average $\operatorname{VAP}(\mu \mathrm{m} / \mathrm{s})$. All phenotypes were assessed after 5 and 90

$139 \mathrm{~min}$ of incubation of the samples at $37^{\circ} \mathrm{C}$, except for sperm concentration, ORT,

140 sperm abnormalities and cytoplasmatic droplets that were measured only after $5 \mathrm{~min}$

141 of incubation of the samples at $37^{\circ} \mathrm{C}$.

142 Sperm phenotypes were then corrected for the fixed effects of farm, season and year

143 of collection and boar age with the "Im" function of R (R Developmental Core Team,

144 2010) using a multiple linear regression model. The $90 \mathrm{~min} / 5$ min incubation ratios

145 were also calculated. In total, 25 phenotypic measures per sample were recorded.

146 Correlations across traits were assessed with the R package "corrplot" (Taiyun and

147 Viliam, 2017).

148 The different analyses are described below, and the complete outline is summarized

149 in Additional Figure 1.

150 Genome Wide Association Study (GWAS)

151 Two hundred and eighty-eight boars were genotyped using the high-density (660K

152 markers) Axiom ${ }^{\mathrm{TM}}$ Porcine Genotyping Array (Thermo Fisher Scientific). The

153 resulting genotype dataset was stringently filtered by excluding these samples with a

154 genotype QC call rate below 96\%. SNP locations were converted from Sscrofa10.2 
155 to Sscrofa11.1 coordinates using plink v1.9 (Purcell et al., 2007). We then excluded

156 SNPs which (i) had a minor allele frequency below 0.05, (ii) deviated from Hardy-

157 Weinberg equilibrium ( $P$-value $<0.001$ ) and (iii) showed above $5 \%$ of missing 158 genotypes. Single-SNP association analysis was carried with the GCTA v.1.91.5 159 software (Yang et al., 2011) considering the genomic relatedness matrix (GRM) as a 160 random effect to correct for the population structure with the following model:

$$
Y_{i j k l}=\mu+S N P_{i}+\text { Farm }_{j}+\text { SeasonYear }_{k}+A g e_{l}+e_{i j k l}
$$

162 where $\left(Y_{i j k l}\right)$ is the phenotype modeled as a function of the population mean $(\mu)$, 163 fixed effect of each SNP $\left(S N P_{i}\right)$, fixed effect of farm $\left(F_{a r m}\right)$, season and year 164 (SeasonYear $\left.{ }_{k}\right)$, age $\left(A g e_{l}\right)$ and a random residual effect $\left(e_{i j k l}\right)$.

165 We adopted a SNP significance threshold of corrected P-values with FDR. 166 Significantly associated SNPs with consecutive distance below 2 Mbp were 167 considered to belong to the same GWAS interval. A new interval was called if the 168 consecutive SNPs were > 2 Mbp apart. SNPs mapping to sexual chromosomes or to 169 unmapped scaffolds were not taken into account. Genetic heritability was assessed 170 with GCTA v.1.91.5 (Yang et al., 2011). Manhattan plots were performed with the 171 “qqman” R package (Turner, 2014).

\section{RNA isolation, sequencing and gene annotation}

173 RNA isolation from 40 sperm samples was performed as previously described

174 (Gòdia et al., 2018b) and included 35 samples from boars analyzed in the GWAS.

175 The other 5 boars did not pass the genotyping quality control and were thus not 176 included in the GWAS. Extracted RNA was subjected to quality control assays 177 including quantification with the Qubitm RNA HS Assay kit (Invitrogen), assessment 178 of RNA integrity with the 2100 Bioanalyzer using the Agilent RNA 6000 Pico kit 179 (Agilent Technologies) and evaluation by RT-qPCR of the sperm-specific PRM1, the 
180 somatic PTPRC mRNA and genomic DNA to confirm that the samples were free

181 from somatic cell RNA and gDNA contaminations.

182 The ribosomal RNA (rRNA) from the 40 RNA samples was depleted with the

183 Ribosomal RNA depletion Kit (Illumina) and libraries were prepared with the

184 SMARTer Low Input Library Prep kit (Clontech) and sequenced to generate $75 \mathrm{bp}$ pair-end reads in an Illumina's HiSeq2000/2500. Undepleted total RNA was also subjected to short noncoding RNA (sncRNA) library preparation (34 of the previous 40 samples) using the NEBNext library prep kit (New England Biolabs) and sequenced at 50 bp single-end in a Hiseq2000 (Illumina).

189 Total RNA-seq reads were evaluated for quality control with FastQC

190 (https://www.bioinformatics.babraham.ac.uk/projects/fastqc/). Low quality reads and sequencing adaptors were trimmed with Trimmomatic v.0.36 (Bolger et al., 2014).

192 Filtered reads were mapped to the porcine genome (Sscrofa 11.1) using HISAT2 v.2.1.0 (Kim et al., 2015). Duplicate reads were removed with Picard Tools v.2.18.29

194 (http://picard.sourceforge.net) Markduplicates. RNA levels of the genes annotated in 195 the porcine genome (Ensembl v.91) were then quantified with StringTie v.1.3.4 196 (Pertea et al., 2015). Only genes with average RNA abundances $\geq 10$ Fragments Per Kilobase of exon per Million reads mapped (FPKM) were kept for further analysis with the aim to discard low abundant genes and spuriously mapped reads.

199 The effect of external variables in gene expression was assessed using the following 200 mixed effect model as in Reverter et al. (Reverter et al., 2005):

$$
Y_{i j k l m n}=\mu+L_{i}+G_{j}+G F_{j k}+G Y S_{j l}+G A_{j m}+G R_{j n}+e_{i j k l m n}
$$

202 where $Y_{i j k l m n}$ represents the log2-transformed FPKM value from the i-th library (40 203 levels), j-th gene (4,120 levels), k-th farm (3 levels), I-th year-season (6 levels), m-th 204 age (3 levels) and $\mathrm{n}$-th assay run (4 levels). Accordingly, $Y_{i j k l m n}$ was modeled as a 
205 function of the fixed effect of library $\left(L_{i}\right)$ and the random effects of gene $\left(G_{j}\right)$, gene

206 by farm $\left(G F_{j k}\right)$, gene by year-season $\left(G Y S_{j l}\right)$, gene by age $\left(G A_{j m}\right)$ and gene by 207 assay run $\left(G R_{j n}\right)$. Random residuals in $e_{i j k l m n}$ were assumed to be independent and identically distributed. Variance component estimates and solutions to the model were obtained using VCE6 (Groeneveld, 1994; ftp://ftp.tzv.fal.de/pub/vce6/doc/vce6manual-3.1-A4.pdf).

211 For the sncRNA-seq data, trimming of adaptors and low quality bases was

212 performed with Cutadapt v1.0 (Martin, 2011). Reads were mapped to the Sus scrofa

213 genome (Sscrofa11.1) with the sRNAtoolbox v.6.17 (Rueda et al., 2015) using

214 default settings and with the porcine miRBase (Kozomara and Griffiths-Jones, 2011)

215 release 21 database. Multi-adjusted read counts were normalized by library size as

216 Counts Per Million (CPM). Only miRNAs with average abundance $>1$ CPM in all the

217 samples were considered. miRNA abundance was stabilized with the log2 218 transformation.

219 The relationship between the 25 phenotypes and each of the log2-stabilized mRNA's and miRNA's abundances were calculated using the Pearson correlation coefficient.

SNP calling from RNA-seq data and Linkage Disequilibrium with GWAS lead

222 SNP

223 Mapped RNA-seq reads of the 35 samples with RNA-seq and genotype data were subjected to SNP calling. Variant calling was performed with SAMtools mpileup and BCFtools v.1.9 (Li et al., 2009). Only SNP variants found in at least 10 samples with minimum Phred quality of 25 and minimum read depth of 10 were kept. The effect of

227 the SNP on protein sequence was predicted with SnpEff v.4.3T (Cingolani et al., 228 2012). The new SNP genotypes were merged to the Axiom genotypes and Linkage 
229 Disequilibrium (LD) $R_{2}$ between GWAS lead SNPs and RNA-seq SNPs was

230 assessed with PLINK v1.9 (Purcell et al., 2007).

\section{Expression GWAS}

232 Expression GWAS (eGWAS) included the 35 samples with RNA-seq and genotype 233 data. The RNA abundances of the detected genes were taken as quantitative traits and tested for association with the genotypes that passed quality control using a linear model. Single-SNP association analysis was carried with the GCTA v.1.91.5 software (Yang et al., 2011), with the following model:

$$
Y_{i}=\mu+S N P_{i}+e_{i}
$$

where $\left(Y_{i}\right)$ is the log2-transformed gene abundance modeled as a function of the population mean $(\mu)$, fixed effect of each SNP $\left(S N P_{i}\right)$, and a random residual effect

$240\left(e_{i}\right)$

241 eGWAS significant associations (FDR $\leq 0.05$ ) were considered only if: (i) the

242 eGWAS associated SNP was also a significant hit (FDR $\leq 0.05)$ in the GWAS for 243 sperm quality phenotypes and (ii) the gene's RNA abundance correlated to the same 244 phenotype as the corresponding GWAS SNP hit.

245 SNP co-association and gene co-abundance analyses

246 For the SNP co-association analysis, GWAS results were used to build an 247 Associated Weight Matrix (AWM) (Fortes et al., 2010; Reverter and Fortes, 2013).

248 The AWM was constructed from two matrices that contained row-wise SNPs and 249 column-wise phenotypes. The first matrix included the P-values of the association 250 between each SNP and the phenotype, and the second matrix corresponded to the 251 SNP z-score standardized additive effect. As live cells with intact plasma membrane 252 are essential for fertilization (Berger et al., 1996; Quintero-Moreno et al., 2004), the 253 percentage of viable spermatozoa at 5 min (VIAB_5) was selected as key phenotype 
and the associated SNPs (P-value $\leq 0.01)$ were included in the AWM. In the next step, the dependency among phenotypes was estimated based on the average number of non-key phenotypes associated (Ap) with these SNPs (P-value $\leq 0.01$ ) $(A p \geq 2)$. Then, SNPs located less than 2,500 bp or more than $1 \mathrm{Mbp}$ from the nearest annotated gene (Ensembl v.91) were kept. The most significant SNP from each annotated gene was kept to build the AWM. The standardized SNP effects across phenotypes were computed and represented using the hierarchical cluster analysis based on Euclidean distance with R package "dendextend" (Galili, 2015). Then, significant gene-gene interactions were assessed to build the SNP Network with the Partial Correlation coefficient with Information Theory (PCIT) algorithm

264 (Reverter and Chan, 2008). PCIT applies first-order partial correlation coefficients together with an information theory approach to identify meaningful gene-gene associations (Reverter and Chan, 2008). Only significant gene co-associations were kept in the SNP Network.

For the RNA co-abundance analysis, significant gene-gene interactions to build the

269 RNA Network were also predicted with PCIT using the stabilized RNA abundances. Interactions between genes and miRNAs were also assessed with PCIT (Reverter and Chan, 2008), and only negative significant correlations were kept.

\section{Integration of SNP and RNA network and network visualization}

273 To obtain a robust gene interaction network, only the pair-wise interactions present

274 in both the SNP and the RNA Networks were kept. The resulting network was named as the Shared Network. In addition, these genes not present in the Shared Network but that presented abundance correlation with $>3$ phenotypes were merged with the

277 Shared Network to create the so-called Final Network. This Final Network also 278 included the interactions between miRNA and mRNA genes. Network visualization 
279 was performed with Cytoscape v3.6 (Shannon et al., 2003) and included information

280 on: (i) the number of phenotypes associated to a gene or miRNA, (ii) the phenotype

281 with highest correlation for each gene, (iii) whether the gene was annotated as a

282 Transcription Factor (TF) or TF co-factor, and (iv) whether the gene was present in

283 the Shared Network or was only found in the Final Network. TF and TF co-factors

284 were extracted from the AnimalTFDB3.0 database (Hu et al., 2019a).

Development of a RNA model and SNP panel for the phenotypic prediction of

sperm quality

The RNA abundance of a subset of genes of the network was used to identify which combination of these was a better predictor of the sperm quality phenotypes. For this, we first extracted 20 genes of the network. These genes were (i) correlated with at least 4 phenotypes, (ii) did not present interactions (edges) between them, (iii) all samples presented RNA abundance levels $>0$ FPKM and (iv) were potentially relevant according to the existing literature. The RSQUARE method from the SAS software was used as an exploratory model building to evaluate all possible subsets of linear regressions using gene abundances and sperm phenotypes and extract the $R_{2}$ magnitude from each prediction. Then, we selected the subset of 10 genes that were most commonly present in all the phenotype models. This subset of common genes was then used for the STEPWISE method from the SAS software, which performs a linear regression analysis for each of the phenotypes to develop a model to predict the phenotype based on gene RNA levels. The model is:

$$
Y_{i}=\text { intercept }_{i}+G P E_{i j}+e_{i j}
$$

301 where $\left(Y_{i j}\right)$ represents the predicted phenotype value from i-th phenotypes (25

302 levels), j-th genes (10 levels). $Y_{i j}$ was modeled as a function of the intercept value 303 for the phenotype (intercept ${ }_{i}$ ), the gene abundance by parameter estimate 
$304\left(G P E_{i j}\right)$ and a residual term $\left(e_{i j}\right)$. We also developed a genome-wide SNP marker

305 panel to identify the polymorphisms that could better predict the phenotypic variance

306 of sperm-related traits. The panel included the lead SNPs from the GWAS and from

307 the eGWAS hits and the GWAS most significant SNP for each of the genes included 308 in the network that also: (i) correlated with at least 4 phenotypes and (ii) were 309 identified in the Shared Network. The proportion of the variance explained by these 310 polymorphisms was assessed with GCTA v.1.91.5 (Yang et al., 2011).

312 Results

313 Phenotype statistics

314 Three hundred ejaculates were phenotyped for 25 sperm quality traits (Table 1).

315 Phenotype correlations (Additional Figure 2) were consistent with their physiological

316 similarities. In general, SNP-based heritabilities (Table 1) were low to moderate with

317 motility related traits displaying higher values. MT_90 was the most heritable trait ( $\mathrm{h}_{2}$ :

318 0.39). On the other side, motility ratios, NABN and VIAB_5 showed nearly null

319 heritability (Table 1). The sperm phenotypes correlated with farm, boar age and

320 Season per Year (Additional file 1) and were thus included as fixed effects in the

321 GWAS model and phenotypes were also corrected for these effects to carry the

322 correlation analysis.

323 [Table 1 appears here]

324 GWAS analysis

325 After quality control, 466,592 SNPs and 276 samples remained for the GWAS. A 326 total of 324 SNPs across autosomal chromosomes and unplaced scaffolds displayed 327 genetic associations (FDR $\leq 0.05)$ with 1 or more sperm quality phenotype (Table 1; 
328 Additional file 2). Of these, 255 SNPs mapped in unplaced scaffolds and were not

329 considered for further data analysis (Additional file 2). A total of 19 chromosomal

330 regions tagged by 69 significant SNPs were identified in Sus scrofa chromosomes

331 (SSC) 1, 3, 4, 6, 7, 9, 13 and 16. The number of SNPs displaying significant

332 associations (FDR $\leq 0.05)$ for each trait is summarized in Table 2.

333 Seven sperm quality traits exhibited significant association signals (Figure 1. A - G;

334 Additional file 2), and only one SNP was associated to more than 1 trait (Table 2;

335 Figure 1. D - E; Additional file 2). HABN and NABN presented the largest number of

336 SNP signals with 41 and 18 associated SNPs, respectively (Figure 1. A and C;

337 Additional file 2). Six of the 19 QTLs were represented by only 1 associated SNP

338 and were discarded from further analyses (Table 2; Figure 1). The most significant

339 SNPs (rs318575212 and rs332927981) of the study were associated to ACRO_5

340 (both with FDR $=0.006$ and Additive effect $=4.11)($ Table 2).

341 [Table 2 appears here]

342 [Figure 1 appears here]

\section{Sperm RNA isolation, RNA-seq and bioinformatics analysis}

344 Isolated RNA from mature spermatozoa was free from somatic cell RNA. Total RNA-

345 seq resulted in an average of $40.7 \mathrm{M}$ reads per sample and $98.2 \%$ of the reads passed the quality control filters (Additional file 3). An average of $83 \%$ of the reads mapped to the porcine genome and after duplicate removal and RNA abundance

348 filters, we identified 4,120 genes (Additional file 4). The Variance Component

349 Estimate mixed model explained $84 \%$ (80\% due to the main effect of gene) of the

350 variation in gene abundance. Consequently, RNA abundances were not corrected 351 for external effects. For short RNA-seq, we obtained an average of 7.3 $\mathrm{M}$ of reads 
352 per sample. Of these, $99.2 \%$ passed quality control and $81.5 \%$ mapped to the 353 porcine genome (Additional file 3). We identified 95 miRNAs out of the 306 that are 354 annotated in swine (Additional file 4).

\section{SNP calling from RNA-seq and Linkage Disequilibrium with GWAS hits}

356 Under the hypothesis that some of the GWAS hits may be tagging a causal variant

357 altering protein sequence and function, and to identify additional SNPs with the potential to be better genetic markers than these identified in the GWAS, we sought to identify variants in annotated genes using the RNA-seq data. As a requisite, these variants had to be in LD with the cognate GWAS hit. After filtering, we identified 7,719 expressed variants, 37 of which mapped within the genomic intervals identified in the GWAS (Table 2; Additional file 5). Twenty-three SNPs were predicted to have low impact effect on protein sequence (synonymous variants and 5' UTR premature start codon), 13 SNPs showed moderate effect (missense variants) and 1 SNP was predicted as a splice donor variant and to have, thus, a high impact on protein sequence (Additional file 5).

SSC13 11 associated to HABN, harbored 21 expressed SNPs (7 and 14 with moderate and low effect, respectively). The polymorphism rs331304027 (a missense variant with moderate effect on the ULK4 gene) was in moderate LD (LD=0.40) with the strongest GWAS SNP hit of the interval (rs690794887) (Table 3). SSC13 I2, also associated to HABN, presented 11 SNPs (1 with high, 5 with moderate and 5 with low effect on protein sequence). Of these, the variant with highest LD ( $L D=0.2)$ with

373 the GWAS hit (rs327865244) was a 5' UTR premature start codon gain (low effect)

374 SNP (rs323872641) in the $A B H D 14 A$ gene (Table 3; Additional file 5). This interval was the only one that presented a SNP with high effect (novel), a splice donor variant in the IQCF5 gene, but the SNP was in low LD (LD=0.02) with the GWAS hit 
377 (Additional file 5). The interval SSC7 12, associated to NABN, encompassed 2 378 expressed SNPs (both with low effect). rs330912302 (a synonymous SNP in the

379 CHD2 gene) presented a moderate $L D(L D=0.4)$ with the strongest hit of the interval 380 (rs336588919) (Table 3). The SSC1 I3 region associated to HABN harbored 3 381 expressed SNPs (1 with moderate and 2 with low effect) (Table 3; Additional file 5).

382 [Table 3 appears here]

383 Correlation of gene's and miRNA's abundances with sperm quality traits

384 The correlation analysis of the 4,120 genes and the 25 phenotypes resulted in 6,128 385 significant correlations ( $P$-value $\leq 0.05$ ) involving 3,007 genes and the 25 traits

386 (Additional file 6). These genes presented between 1 and 9 significant correlations

387 with the different semen quality traits (Additional file 6). 344 genes were significantly 388 correlated with $\geq 4$ traits. For the miRNAs, the abundance of the 95 miRNAs and the 389 studied phenotypes resulted in 306 significant correlations ( $P$-value $\leq 0.05)$ which 390 involved 87 miRNAs and 17 semen traits (Additional file 7). The miRNAs presented

391 between 1 and 9 significant correlations with the semen quality traits studied 392 (Additional file 7).

\section{Expression GWAS analysis}

394 In order to predict whether the GWAS hits were tagging a causal variant altering gene expression we carried an eGWAS. eGWAS was performed with the genotypes of 464,020 SNPs that passed the quality control and the normalized RNA

397 abundances. We then only focused on the associations between GWAS SNP hits 398 (with FDR $\leq 0.05$ ) and transcripts which abundances correlated with the same 399 phenotype. We identified 45 SNPs (FDR $\leq 0.05$ ) located in 3 genomic regions 400 related to ACRO_5 and HABN (Table 4). Six SNPs had unknown positions in the 
401 genome after liftover from Sscrofa10.2 to Sscrofa11.1. The remaining eGWAS hits

402 were in SSC4, 6 and 13 (Table 4; Additional file 8). All the SNPs had a trans effect,

403 related to genes located in different chromosomes. The eQTL identified in SSC4,

404 was related to ACRO_5 and was associated to 3 genes, NCLN, ASCC1 and AATF.

405 Also involving ACRO_5, the eQTL in SSC6 was associated to the IQCJ gene.

406 Finally, the eQTL in SSC13 for HABN, included SNPs associated to HARS, ACTR2,

407 EPB41L3 and RAB1B.

408 [Table 4 appears here]

\section{Gene network analysis}

410 After SNP selection, 2,648 of the 466,592 SNPs were retained to build the AWM.

411 Trait hierarchical cluster distributions were in agreement with the biological

412 similarities and phenotypic correlations (Additional Figure 2 and 3). A clear

413 separation between (i) morphological abnormalities and motility parameters and (ii)

414 cell viability and ORT was observed based on the additive effects of the SNPs

415 calculated in the association analysis. In keeping with previous studies (Ramayo-

416 Caldas et al., 2016; Snelling et al., 2013), the SNPs detected with the AWM

417 explained $74.1 \%$ of the phenotypic variance of the key phenotype (VIAB_5). The

418 SNP network predicted with PCIT (Reverter and Chan, 2008) resulted in significant

419 correlations involving 2,648 nodes (all the genes) connected by $2,984,616$ edges

420 (Table 5). 
425 Table 5. Number of nodes (genes) and edges (interactions).

\begin{tabular}{llll}
\hline Network & Nodes & Edges & Observations \\
\hline SNP Network & 2,648 & $2,984,616$ & \\
RNA Network & 4,120 & $1,173,995$ & \\
Shared Network & 613 & 16,591 & \\
& & & \\
Final Network & & & \\
miRNAs & 94 & 1,564 & \\
Protein coding genes & 1,313 & 81,733 & \\
& 1,135 & & Correlated with > 1 phenotype \\
& 68 & & TF \\
& 89 & & TF co-factor \\
\hline
\end{tabular}

$426 \quad \mathrm{TF}=$ Transcription Factor

427

428 For the RNA network analysis, the RNA levels of the 4,120 detected genes were 429 used to identify potential connections with PCIT (Reverter and Chan, 2008). The 430 RNA network included 4,120 nodes (all the genes) connected by $1,173,995$ edges 431 (Table 5). PCIT also built 4,539 significant interactions between 95 miRNAs and 630 432 genes.

433 To obtain the Shared Network, common SNP and RNA network edges were 434 extracted, thus, focusing only in the shared set of interacting genes from both 435 approaches. This comparison resulted in 613 nodes connected by 16,591 edges 436 (Table 5). The Final Network included a set of 700 additional genes (as they 437 correlated with $>3$ phenotypes) and their interactions. Moreover, the Final Network 438 also involved 1,564 edges connecting 202 genes and 94 miRNAs (Table 5). Of the 4391,313 genes included in the Final Network, the abundance of 1,135 correlated with at 
440 least 1 phenotype, 68 have been reported as TFs and 89 as TFcos (Figure 2.A;

441 Table 5). Nearly a quarter of the genes (282 out of the 1,313) presented at least 200

442 edges. The genes that presented more interactions were PLCH2 (579 edges,

443 present in the Final but not in Shared Network and correlated with 3 phenotypes),

444 CEP152 (399 edges, in the Shared Network and correlated with 4 traits) and 445 SLC41A2 (382 edges, in the Shared Network).

446 Gene ontology analysis of the genes included in the Final Network presented

447 enrichment for DNA repair (e.g. RAD51, SETX, SOD1), meiotic cell cycle (e.g.

448 BAG6, HSPA2, RAD51), gamete generation (e.g. TSSK3, PRDM14, PRKAR1A) and

449 spermatogenesis (e.g. BAG6, CAPZA3, HSPA2) (Additional file 9).

450 Development of a RNA model and a SNP panel

451 The $\mathrm{R}_{2}$ model predicted that the RNA levels of 20 genes could explain between 55 to $45278 \%$ of the phenotypic variation across traits. The selection of 10 genes that were 453 most commonly present in all the phenotype models explained the vast majority (93 454 to $99 \%$ ) of the phenotypic variation that was predicted by the model. The final set of 45510 genes included in the linear regression model was: MICAL3, EFHC1, TRAPPC2L, 456 ATP9A, THADA, MOBKL3, BLVRB, LARP4, CARS2 and NDUFV2. The analysis 457 resulted in significant models for 10 of the 25 phenotypes (Table 6 ). The most 458 significant model was for PDROP, which could predict the phenotype with an 459 efficiency of $68 \%$ (Table 6). The estimated parameters of the significant models can 460 be found in Additional file 10. 
464 Table 6. R2 and phenotypic variance for each trait from the RNA model and SNP 465 panel.

\begin{tabular}{|c|c|c|c|}
\hline \multirow[t]{2}{*}{ Acronym } & \multicolumn{2}{|c|}{ RNA model } & \multirow{2}{*}{$\begin{array}{c}\text { SNP panel } \\
\text { Phenotipic variance explained } \\
\text { (SE) }\end{array}$} \\
\hline & $\mathbf{R}_{2}$ & P-value & \\
\hline CON & 0.17 & 0.82 & $0.05(0.05)$ \\
\hline VIAB_5 & 0.43 & 0.06 & $0.27(0.07)$ \\
\hline VIAB_90 & 0.23 & 0.61 & $0.28(0.07)$ \\
\hline ORT & 0.22 & 0.62 & $0.24(0.07)$ \\
\hline HABN & 0.16 & 0.84 & $0.29(0.06)$ \\
\hline NABN & 0.22 & 0.64 & $0.36(0.07)$ \\
\hline TABN & 0.26 & 0.49 & $0.26(0.07)$ \\
\hline PDROP & 0.68 & $<0.0001$ & $0.17(0.07)$ \\
\hline DDROP & 0.42 & 0.07 & $0.06(0.05)$ \\
\hline MT_5 & 0.46 & 0.03 & $0.31(0.07)$ \\
\hline MT_90 & 0.34 & 0.22 & $0.30(0.07)$ \\
\hline VAP_5 & 0.58 & 0.002 & $0.34(0.07)$ \\
\hline VAP_90 & 0.55 & 0.005 & $0.34(0.07)$ \\
\hline VCL_5 & 0.61 & 0.001 & $0.33(0.07)$ \\
\hline VCL_90 & 0.55 & 0.01 & $0.34(0.07)$ \\
\hline VSL_5 & 0.36 & 0.16 & $0.31(0.07)$ \\
\hline VSL_90 & 0.61 & 0.001 & $0.33(0.07)$ \\
\hline ACRO_5 & 0.5 & 0.02 & $0.21(0.06)$ \\
\hline ACRO_90 & 0.21 & 0.68 & $0.23(0.07)$ \\
\hline R_MT & 0.3 & 0.35 & $0.13(0.06)$ \\
\hline R_VAP & 0.18 & 0.79 & $0.18(0.07)$ \\
\hline R_VCL & 0.28 & 0.42 & $0.14(0.07)$ \\
\hline R_VSL & 0.21 & 0.68 & $0.21(0.07)$ \\
\hline R_VIAB & 0.44 & 0.05 & $0.23(0.07)$ \\
\hline R_ACRO & 0.57 & 0.003 & $0.19(0.07)$ \\
\hline
\end{tabular}

466 Acronym descriptions can be found in Table 1. SE: Standard Error 
468 The SNP-based panel was built with 73 polymorphisms (18 lead SNPs from GWAS 469 hits, 2 lead SNPs from the eGWAS hits, 53 SNPs from the Shared Network and

470 correlated $\geq 4$ phenotypes) (Additional file 11). These polymorphisms could explain 471 between 5 to $36 \%$ of the phenotypic variance across the 25 traits (Table 6 ). A 472 moderate proportion ( $>20 \%$ ) of the phenotypic variance could be explained for 18 of 473 the 25 traits. The best predictions were for sperm abnormalities (NABN, HABN, 474 TABN) and sperm motility related traits (e.g. MT_5, VAP_90 and VCL_90) (Table 6).

\section{Discussion}

\section{GWAS analysis}

478 Investigating the genomic regions and molecular processes controlling sperm quality

479 has become a focus of interest in human and in livestock including swine, in this 480 case for its relevance on the sustainability of pig breeding and production (Diniz et 481 al., 2014; Gao et al., 2019; Marques et al., 2018; Zhao et al., 2020; Zhao et al., 482 2016). In fact, our results as well as data obtained by other groups (Diniz et al., 483 2014; Marques et al., 2018; Smital et al., 2005; Wolf, 2009), have shown that boar 484 sperm quality has a genetic basis and that it can thus be selected for breeding 485 strategies.

486 The GWAS revealed 12 QTL regions represented by 2 or more significant SNPs and 487 several positional candidate genes for HABN, NABN, ACRO_5 and MT_5 (Table 2). 488 The highest signals were on SSC4 for ACRO_5 ( 2.41-2.42 Mbp) (Table 2; 489 Additional file 2), $\sim 69 \mathrm{~kb}$ upstream of the Solute Carrier Family 45 Member 4 490 (SLC45A4) gene. SLC45A4 encodes a proton-coupled sugar transporter implicated 
491 in the nutrition of spermatozoa during their maturation in epididymis (Vitavska and

492 Wieczorek, 2017) where acrosome assembly continues its posttesticular sperm 493 maturation (Olson et al., 2003). The Solute Carrier Family 35 Member B3 (SLC35B3) 494 was selected as a potential candidate for the MT_5 QTL on SSC7 (Table 2; 495 Additional file 2). SLC35B3 maps 0.6 Mbp away from this QTL. Although a role in sperm has not been reported thus far, the SLC35 gene family has been postulated to 497 play a role as nucleotide sugar transporter (Song, 2013) and we propose that it may also be relevant for the nutritional support of spermatozoa.

We detected several significant regions for HABN (Table 2; Additional file 2). The QTL on SSC1 12 ( 94.9-98.8 Mbp) included interesting candidate genes such as the

501 Katanin Catalytic Subunit A1 Like 2 (KATNAL2). Dunleavy et al. (Dunleavy et al., 502 2017) reported that Katnal2 is a critical regulator of male germ cell development affecting sperm head shaping, acrosome attachment and sperm tail growth. Other candidate genes in that region were the solute carrier SLC14A2, encoding the urea transporter A, suggested to participate in sperm head formation by reducing its

506 volume though excreting urea (Li et al., 2012), or the SMAD Family Member 2 507 (SMAD2) involved in spermatogonial differentiation (Wu et al., 2017). On SSC13 I1, 508 we identified two candidate genes: the Testis and Ovary-specific PAZ domain gene 1 509 (TOPAZ1) and the IQ Motif Containing F1 (IQCF1). Luangpraseuth-Prosper et al. 510 (Luangpraseuth-Prosper et al., 2015) demonstrated that Topaz1 knockout mice 511 presented meiotic arrest and caused male infertility. As for IQCF1, Fang et al. (Fang 512 et al., 2015) reported that this gene localizes in the acrosome and that it is involved 513 in sperm capacitation in mice. Iqcf1-/- mice were significantly less fertile than wild 514 type mice (Fang et al., 2015). The QTL region on SSC13 I2 included the candidate 515 Protein Kinase C Delta $(P R K C D)$ gene. PRKCD has been involved in 
516 spermatogenesis and embryonic development (Suh et al., 2003) and was highlighted

517 in a GWAS for semen volume in Holstein-Friesian bulls (Hering et al., 2014).

518 Four QTL regions were identified for NABN (Table 2; Additional file 2). The QTL on

519 SSC1 15 included as a candidate gene the transporter ATP Binding Cassette

520 Subfamily A Member $1(A B C A 1)$. In humans, ABCA1 localizes in the dorsal side of

521 the sperm head and in the middle piece of the tail (Morales et al., 2008). It has been

522 suggested to contribute to cholesterol transport and fertilization capacity (Morales et

523 al., 2008). The QTL in SSC7 12 included two genes of interest, the Chromodomain

524 Helicase DNA-binding protein 2 (CHD2) and the Sialyltransferase 2 (ST8SIA2).

525 CHD2 may be playing an important role in DNA damage response and genome

526 stability maintenance (Nagarajan et al., 2009). In humans, CHD2 has been

527 associated with non-obstructive azoospermia (Qin et al., 2014). Simon et al. (Simon

528 et al., 2013) demonstrated that the protein encoded by ST8SIA2 is located in the

529 post-acrosomal region of human sperm. It generates polysialic acid, which is

530 suggested to act as a cytoprotective element to increase the number of viable sperm

531 (Simon et al., 2013).

532 Four of our GWAS hits map near previously reported QTLs for semen quality traits.

533 This is the case for the SSC1 I6 QTL, associated to NABN, which mapped $335 \mathrm{kbp}$

534 downstream from a QTL associated to sperm abnormalities and motility in boars

535 (Marques et al., 2018). The QTL SSC3 12, associated to NABN lies $350 \mathrm{kpb}$

536 upstream from a PDROP QTL (Zhao et al., 2020). The SSC4 I1 QTL, associated to

537 ACRO_5, resides 655 kpb upstream from a QTL for the Distal Midpiece Reflex (Zhao

538 et al., 2020) and the SSC7 I1 QTL, associated to MT_5 maps 123 kpb upstream

539 from a PDROP QTL (Zhao et al., 2020). The discrepancies across studies could

540 arise due to different technical (e.g. sample size, SNP arrays, QTL or phenotyping 
541 accuracy), environmental (e.g. temperature, animal husbandry or sperm processing)

542 or biological factors (e.g. genetic heterogeneity).

\section{SNP calling from RNA-seq data}

545 Calling genomic variants from RNA-seq data can be a complementary method to

546 detect previously unknown or ungenotyped polymorphisms in transcribed genes that

547 might carry important functional implications or may be better genetic markers for

548 that given trait. Should these genes be involved in related phenotypes and these

549 variants be: (i) in LD with the GWAS lead SNP and (ii) have a predicted effect on

550 protein sequence, these polymorphisms could be suggested as potential causal

551 candidates. For that purpose, we sought to identify transcribed variants in the QTL

552 regions and assessed their LD with the lead SNP hit of the QTL.

553 For HABN we found new genetic variants in genes of physiological interest (Table 3;

554 Additional file 5). On SSC13 I1, we discovered several variants in the Unc-51 Like Kinase 4 (ULK4) gene in moderate LD with the lead SNP of this GWAS hit (Table 3; Additional file 5). Although ULK4 has not been associated to sperm defects, Liu et al.

557 (Liu et al., 2016) showed that this gene has an essential role in ciliogenesis, the 558 process of formation of cilium or flagellum, a microtubular structure located in the center of all motile cilia and flagella, also in sperm. In fact, the disruption of another ciliogenesis-related gene (Ift25) has resulted in infertile males with round sperm

561 heads and abnormal tails (Liu et al., 2017). On SSC13 I2 we identified one variant

562 with predicted high effect in the IQCF5 gene (Additional file 5). The IQ Motif family of

563 proteins have been reported in myosins and promote calcium regulation (Bahler and 564 Rhoads, 2002). Myosins are actin-based motors that translocate along actin 
565 filaments in an ATP-depending manner and have been implicated in various aspects

566 of spermatogenesis (Hu et al., 2019b). In sperm, actin filaments are located in the

567 acrosomal region (Breitbart et al., 2005). Interestingly, the previously discussed

568 GWAS positional and physiological candidate genes CHD2 and KATNAL2, also

569 presented genetic variants in LD with the lead SNPs at SSC7 12 (low effects:

570 rs330912302 LD $=0.4$ and rs339719658 LD $=0.37$ ) and SSC1 I3 (low effects:

571 rs700749617 LD $=0.01$, rs710447566 LD $=0.07$ or moderate effect: $r$ s690151450

572 LD $=6.9 \times 10-3$ ), respectively (Additional file 5).

\section{Correlation between genes and miRNAs with semen traits}

574 For mRNA transcripts, the strongest correlation was for TTC28 and HABN $(-0.71)$

575 (Additional file 6). TTC28 is required for the condensation of spindle microtubules

576 during mitosis and meiosis (Izumiyama et al., 2012). Other genes of interest included

$577 \quad A B C A 3$, which RNA levels correlated with 9 phenotypes (Additional file 6). This gene

578 is an $\mathrm{ABC}$ transporter that plays a role in flipin-cholesterol complexes as a 579 mechanism to remove cholesterol from the sperm membrane (Mengerink and

580 Vacquier, 2002). Although the molecular basis induced by cholesterol efflux from

581 sperm is not well understood, it has been reported to be required for sperm 582 capacitation (Visconti et al., 2002). Another example is EFHC1, which RNA levels 583 correlated with 6 phenotypes (Additional file 6). Efhc1-/ knockout mice presented 584 reduced flagellar beating frequency (Suzuki et al., 2009).

585 Several miRNAs of interest including miR-23a, miR-27a and miR-122 correlated with

5867,8 and 8 semen quality traits, respectively (Additional file 7). miR-23a, has been

587 found to be dysregulated in men's subfertility (Abu-Halima et al., 2019). miR-27a

588 abundance of spermatozoa has been related to lower progressive motility and 589 normal morphology (Zhou et al., 2017). miR-122 expression was associated with 
590 abnormal sperm development (Liu et al., 2013) and dysregulated in subfertile men

591 (Abu-Halima et al., 2013).

592

593

\section{eGWAS}

594 We also performed a within-trait eGWAS linking for each phenotype, GWAS lead

595 SNPs with genes which RNA abundance correlated with the same trait. These

596 GWAS regions could be tagging causal variants with regulatory functions on gene 597 expression. We identified 3 eQTLs all with a trans-effect (Table 4; Additional file 8).

598 The trans-eQTL on SSC6 was correlated with the abundance of the IQ Motif

599 Containing J (IQCJ) gene, both SNP and mRNA were associated to ACRO_5 (Table

600 4). IQCJ is a member of the previously discussed IQ Motif family proteins. Although it

601 has not been studied in sperm, Martin et al. (Martin et al., 2008) reported the 602 presence of the IQCJ-SCHIP-1 isoform in mammalian neurons and its role in calcium

603 mediated responses. We hypothesize that IQCJ may also mediate calcium response

604 in sperm. In fact, calcium has been involved in the regulation of motility,

605 hyperactivation, capacitation and acrosome reaction (reviewed in: Sun et al., 2017).

606 The trans-eQTL on SSC13 for HABN was associated to several genes including the

607 Actin Related Protein 2 (ACTR2) and Histidyl-TRNA Synthetase (HARS) (Table 4;

608 Additional file 8). Heid et al. (Heid et al., 2002) identified ACTR2 in bull sperm head

609 and suggested that it serves for sperm capacitation and acrosome reaction. On the

610 other side, HARS has been involved in attaching histidines to its corresponding tRNA

611 molecules, a fundamental cellular process for the translation of mRNA into protein

612 (Ibba and Söll, 2000). Waldron et al. (Waldron et al., 2019) showed that HARS

613 zebrafish knockout presented severe defects in high proliferative cells. Although its 
614 role in sperm remains to be resolved, HARS protein has been found overexpressed

615 in sperm of low-fertility bulls (Aslam et al., 2019) and we do not rule out a potential

616 involvement of this gene in spermatogenesis. trans-eQTL hotspots (these trans-

617 eQTLS involving several genes) are of particular interest as their SNPs could harbor

618 important regulatory roles and variations influencing gene expression and thus are

619 more likely to contribute to the phenotype.

\section{Gene network analysis}

621 Despite the considerable number of candidate genes identified in our GWAS, many

622 genes might have been missed by this traditional single-trait approach due to the

623 lack of an acceptable significant association (FDR $>0.05$ ). After all, sperm quality is

624 a complex phenotype influenced by many factors, such as genetics, husbandry,

625 environment, or testicular pathologies that contribute to an intricate network of genes

626 and molecular processes. Moreover, many of the SNPs included in the GWAS may

627 not be at sufficiently high allelic frequency or be in strong LD with the causal variants

628 in the studied populations. An alternative strategy to exploit GWAS information is to

629 perform an AWM analysis that extracts SNPs that while having strong yet below the

630 significance threshold of genetic association, are also associated to a certain number

631 of traits (Fortes et al., 2010). The association of 1 SNP to more than 1 trait provides

632 additional robustness to the potential relevance of that SNP to semen quality in our

633 case. This, followed by a PCIT analysis to study gene-gene interactions can provide

634 information on the relevant genes and pathways for certain phenotypes and then

635 search for SNPs in or affecting them. Obviously, transcriptomics data can contribute

636 additional valuable information in the description of these genes and pathways. The

637 integration of both sources of information can also be used to improve the accuracy

638 of genomic predictions (Ramayo-Caldas et al., 2019). For this reason, we have 
639 addressed the genetics behind boar's sperm quality through an integrative systems

640 biology approach. The genetic co-association and RNA co-abundance interactions

641 revealed a number of appealing features such as new candidate genes, TFs, TF-cos

642 and miRNAs that belong to biological processes and relevant functions related to

643 sperm.

644 The TF with the highest number of predicted interactions (129) was encoded by the 645 Calcium Responsive Transcription Factor (CARF) gene, which RNA abundance was 646 in turn, correlated with 9 phenotypes (Figure 2.B; Additional file 6). CARF acts as a 647 transcriptional activator promoted by calcium influx (Tao et al., 2002). Since calcium 648 ions are essential in sperm function (Publicover et al., 2007), we cannot discard the 649 possibility that this TF could be involved in pathways related to sperm maintenance 650 and functioning. Some of the CARF predicted target genes from our analysis include 651 interesting candidates such as La Ribonucleoprotein Domain Family Member 4 652 (LARP4), THADA Armadillo Repeat Containing (THADA) and EF-Hand Domain 653 Containing 1 (EFHC1) gene. LARP4, has been proposed to regulate mRNA stability 654 and translation of mRNAs (Blagden et al., 2009). Blagden et al. (Blagden et al., 655 2009) reported Drosophila larp knockout mutants resulted in a considerable 656 proportion of spermatocytes with meiotic defects. Although the role of THADA 657 remains uncertain in sperm, Moraru et al. (Moraru et al., 2017) showed that in 658 Drosophila, THADA modulates calcium signaling, energy storage and thermogenesis 659 balance. EFHC1 encodes for a myoclonin1 protein and has been detected in sperm 660 flagella in mice testis (Suzuki et al., 2008). Although Efhc1-deficient mice were 661 fertile, mutants presented a reduced ciliary (flagellar) beating frequency (Suzuki et 662 al., 2009). 
663 Other TFs with a large number of interactions were the SMAD Family Member 4 664 (SMAD4) gene (interacting with 32 genes) and the Lysine Demethylase 3A (KDM3A) 665 gene (281 gene interactions), both potentially targeting a set of genes enriched for 666 cellular macromolecular complex assembly processes (Additional file 9). TFs 667 involved in DNA repair, such as the Bromodomain Adjacent To Zinc Finger Domain 668 1B $(B A Z 1 B)$, were also identified. Its closest paralog, $B A Z 1 A$ encodes a member of 669 the chromatin remodeling complex (Racki et al., 2009). Dowdle et al. (Dowdle et al., 670 2013) showed that Baz1a-- mice were infertile because of spermatogenesis defects 671 tied to changes in chromatin composition. Another TF of interest was the Estrogen 672 Receptor 1 (ESR1), which was present in the shared network. ESR1 has been already associated with pig sperm motility and cytoplasmatic droplets (Gunawan et al., 2011). Moreover, polymorphisms in ESR1 have been suggested to influence estrogen levels which in turn, affect sperm motility (Carreau et al., 2002).

676 The network comprised new candidate genes for sperm quality. The Trafficking 677 Protein Particle Complex 2 Like (TRAPPC2L) gene, correlated with 27 miRNAs 678 including miR-30d, a miRNA that was dysregulated in oligozoospermic infertile 679 individuals (Salas-Huetos et al., 2015) (Figure 2.C). TRAPPC2L belongs to the 680 TRAPPC family, with a reported role in ciliogeneisis (Westlake et al., 2011). 681 Interestingly, TRAPPC2L was found associated in the Final Network with the 682 Spermatogenesis And Centriole Associated 1 (SPATC1) gene, a gene that has been 683 localized in the neck region of the mouse and human sperm (Goto et al., 2010). 684 Disruption of its homolog Spatc1/ in mice led to male sterility due to separation of 685 sperm heads from tails, thereby advocating for a role in sperm head-tail integrity 686 (Kim et al., 2018). The network also included DNAI2, which correlated with 4 687 phenotypes (Additional file 6). Mutations in DNAl2 have been associated with ciliary 
688 defects and with males showing reduced fertility due to impaired sperm tail function

689 (Loges et al., 2008). DNAI2 has been related to boar sperm motility in a previous

690 GWAS (Marques et al., 2018). CHD2 is another interesting gene in the network as it 691 was also identified as a candidate gene in our GWAS analysis. This gene also 692 presented new DNA variants in LD with GWAS lead SNPs which would be worth 693 testing in a genetic association study (Figure 2.D; Table 3). CHD2 was 694 hidroxymethylated in human sperm after exposure to bisphenol A, an epigenetic 695 modifier that causes spermatogenesis defects and alters sperm motility (Zheng et 696 al., 2017).

697 Of the 94 miRNAs identified in sperm and included in the final network, 30 interacted 698 with at least 20 genes. Some of these 30 miRNAs correlated with sperm traits and 699 have also been previously linked to sperm quality and fertility in other studies. 700 Noteworthy, miR-16, a miRNA that was down-regulated in the semen of infertile 701 males with sperm abnormalities (Liu et al., 2012), correlated with 4 sperm 702 phenotypes (Additional file 7) and potentially interacted with 67 genes (e.g. ATP9A, 703 found in the Shared Network and included in the RNA model). Similarly, miR-10b, 704 previously associated with human infertile semen samples (Tian et al., 2017), 705 correlated with a motility-related parameter (VCL) and interacted with 32 genes 706 (including the previously discussed TRAPPC2L that is present in the Final Network).

\section{Development of a RNA and a SNP models}

708 In this study, we provide a novel and innovative approach to develop a RNA model

709 to estimate the phenotypes based on gene abundances. The model, including 10 710 genes, was predicted to be significant for 10 phenotypes and performed best for 711 PDROP and some of the motility related traits (Table 6). The model for PDROP 712 reported a highly significant role of the THADA gene (Additional file 10), which at the 
713 same time was present in the Shared Network and its RNA levels are positively

714 correlated with PDROP. THADA regulates the metabolism via calcium signaling by

715 binding the sarco/ER Ca2+ ATPase transporter mechanism (Harper et al., 2005). The

716 CARS2 gene was also a strong contributor in the model for PDROP and was also

717 identified in the Shared Network (Additional file 10). This gene has a critical role in

718 protein synthesis but no direct link to spermatogenesis or sperm function has been

719 reported.

720 Although SNPs have become the marker of choice for the genetic improvement of

721 livestock species, the development of a SNP array for the prediction of the boar's

722 sperm quality remains to be done. Here, we propose a SNP model with 73 SNPs

723 including the polymorphisms identified through the GWAS, eGWAS and gene : gene

724 interaction and phenotypic correlation analysis (Additional file 11). The model could

725 hold promising potential for its application in animal breeding programs. This panel of

72673 SNPs could estimate between 5 to $36 \%$ of the phenotypic variance across the 25

727 traits that were evaluated. These SNPs were better predictors for the phenotypes

728 related to sperm abnormalities and motility (Table 6). Remarkably, when only

729 considering the GWAS lead SNPs, the panel explained between 4 to $26 \%$ of the

730 phenotypic variance, and only for 3 traits (HABN, NABN and TABN) the model would

731 be able to predict above $20 \%$ of the phenotypic variance. Thus, this systems biology

732 approach allowed including an additional set of SNPs that increased the predictive

733 potential of the panel.

734 In a previous study for sperm motility and morphological abnormalities using two 735 porcine lines, Marques et al. identified several QTLs that cumulatively explained $73610.8 \%$ of the genetic variance (Marques et al., 2018) including 412 and 271 SNPs for 737 each line. Gao et al. (Gao et al., 2019) identified 20 and 16 QTLs that could explain 
$73835.3 \%$ and $20.6 \%$ of sperm motility and morphological abnormalities traits in Duroc

739 boars, respectively. Our approach was able to predict $30-31 \%$ and $26-36 \%$ of the

740 variance of the same group of traits with only 73 SNPs for motility and

741 morphological-related traits, respectively (Table 6). However, we have employed an

742 integrated and informed approach based not only on the GWAS and eGWAS FDR

743 significant associations but also in a robust network built from co-associated SNPs

744 (identified at suggestive levels but across several phenotypes) as well as gene RNA co-abundance. Moreover, our SNPs were chosen to minimize LD between them and thus maximize the informativity of the panel. This allowed the informed inclusion of a

747 large number of SNPs with independent marker potential and thus the development

748 of a more powerful panel for the prediction of semen quality in pigs.

749 Although the results only hold in our population and the validation of the panel will

750 require additional evaluations in other populations, the integrative approach

751 proposed in this study to ultimately build a SNP array provides compelling results of

752 its application to any type of complex trait with a genetic basis. This offers another

753 avenue to improve traits influenced by several genes that are of interest for the 754 animal breeding industry.

\section{Conclusions}

757 In summary, our results suggest that genetic variants identified in the 12 QTL 758 regions mapped to - or near - CHD2, KATNAL2, SLC14A2, IQCF1 and ABCA1,

759 together with other candidate genes based on a systems biology approach including 760 among others, LAPR4, THADA, EFHC1, SMADA4, SPATC1 or TRAPPC2L, may modulate sperm quality in pigs. This network also includes TFs such as CARF, with 
762 a large number of potential interactions with target genes that are likely to be key

763 players in shaping the complex inheritance of the sperm quality traits. We have

764 developed a DNA marker panel based on a systems biology approach that may be

765 able to explain higher phenotypic variance than what could have been found from a

766 stand-alone GWAS. The model included GWAS lead SNPs, top eGWAS SNPs and

767 SNPs from genes identified in the Shared Network and could potentially explain over

$76830 \%$ of the phenotypic variance of sperm quality traits such as motility and

769 morphology. Although our results are considerably promising for the improvement of

770 the sector, caution should be taken due to the sample size of our study. Further work

771 should include the validation of the RNA and SNP model in a large number of pigs

772 belonging to different breeds and populations. The implications of this research are

773 broad, ranging from applications to animal breeding strategies to modeling the

774 biology of infertility in mammals.

775

776 List of abbreviations

777 ACRO: abnormal acrosomes

778 Al: Artificial Insemination

779 Ap: Associated Phenotype

780 AWM: Associated Weight Matrix

781 CASA: computer-assisted semen analysis

782 circRNA: circular RNA

783 CON: concentration

784 CPM: counts per million 
785 DDROP: distal droplet

786 eGWAS: Expression GWAS

787 FPKM: Fragments Per Kilobase of exon per million reads mapped

788 GWAS: Genome Wide Association Study

789 HABN: head sperm abnormalities

790 LD: Linkage Disequilibrium

791 miRNA: micro RNA

792 MT: percentage of motile cells

793 NABN: neck sperm abnormalities

794 ORT: osmotic resistance test

795 PCIT: Partial Correlation coefficient with Information Theory

796 PDROP: proximal droplet

797 piRNA: Piwi interacting RNA

798 QTL: Quantitative Trait Loci

799 rRNA: ribosomal RNA

800 RT-qPCR: quantitative real time PCR

801 sncRNA: short non-coding RNA

802 TABN: tail sperm abnormalities

803 TF: Transcription Factor

804 VAP: Average Path Velocity

805 VCL: Curvilinear Velocity 
806 VIAB: cell viability

807 VSL: Straight Line Velocity

808

809 Declarations

810 Ethics approval and consent to participate

811 The ejaculates obtained from pigs were privately owned for non-research purposes.

812 The owners provided consent for the use of these samples for research. Specialized

813 professionals at the farm obtained all the ejaculates and blood following standard

814 routine monitoring procedures and relevant guidelines.

\section{Consent for publication}

816 Not applicable

817 Availability of data and material

818 The datasets generated and/or analysed during the current study are available at

819 NCBI's BioProject PRJNA520978. The phenotypic and genotypic datasets used in

820 the current study are available from the corresponding author on reasonable request.

821 Competing interests

822 The authors declare that they have no competing interests.

823 Funding

824 This work was supported by the Spanish Ministry of Economy and Competitiveness

825 (MINECO) under grant AGL2013-44978-R and grant AGL2017-86946-R and by the

826 CERCA Programme/Generalitat de Catalunya. AGL2017-86946-R was also funded

827 by the Spanish State Research Agency (AEI) and the European Regional 
828 Development Fund (ERDF). We thank the Agency for Management of University and

829 Research Grants (AGAUR) of the Generalitat de Catalunya (Grant Numbers 2014

830 SGR 1528 and 2017 SGR 1060). We also acknowledge the support of the Spanish

831 Ministry of Economy and Competitivity for the Center of Excellence Severo Ochoa

832 2016-2019 (Grant Number SEV-2015-0533) grant awarded to the Centre for

833 Research in Agricultural Genomics (CRAG). MG acknowledges a Ph.D. studentship

834 from MINECO (Grant Number BES-2014-070560) and a Short-Stay fellowship from

835 MINECO (EEBB-I-18-12860) at AR's group.

\section{Authors' contributions}

837 MG, AS, and AIC conceived and designed the experiments. JR-G carried the 838 phenotypic analysis. MG performed sperm purifications and RNA extractions. AnC 839 designed and carried the RT-qPCR and their analyses. MG analyzed the data with 840 support from AR, RGP and YRC. MG and AIC wrote the manuscript. All authors 841 discussed the data and read and approved the contents of the manuscript.

\section{Acknowledgements}

843 We thank Betlem Cabrera (CRAG), Dr. Fabiana Quoos Mayer (Instituto de

844 Pesquisas Veterinárias Desidério Finamor) and Dr. Martina Rocco (CRAG) for their 845 laboratory support. We gratefully acknowledge Craig Lewis from Genus PIC and 846 Sam Balasch from Gepork for contributing the sperm samples.

\section{References}

848 Ablondi M, Gòdia M, Rodríguez-Gil JE, Sánchez A, Clop A. Characterisation of 849 sperm piRNAs and their correlation with semen quality traits in swine. bioRxiv. 2020.03.16.994178 
851 Abu-Halima M, Hammadeh M, Schmitt J, Leidinger P, Keller A et al. Altered microRNA expression profiles of human spermatozoa in patients with different spermatogenic impairments. Fertil Steril. 2013;99:1249-55 e16.

Abu-Halima M, Ayesh BM, Hart M, Alles J, Fischer U et al. Differential expression of miR-23a/b-3p and its target genes in male patients with subfertility. Fertil Steril. 2019;112:323-35 e2.

Aslam MKM, Kumaresan A, Yadav S, Mohanty TK and Datta TK. Comparative proteomic analysis of high- and low-fertile buffalo bull spermatozoa for identification of fertility-associated proteins. Reprod Domest Anim. 2019;54:786-94.

861 Bahler $M$, and Rhoads $A$. Calmodulin signaling via the $I Q$ motif. FEBS Lett. 2002;513:107-13.

Berger T, Anderson DL and Penedo MCT. Porcine sperm fertilizing potential in relationship to sperm functional capacities. Anim Reprod Sci. 1996;44:231-9.

Blagden SP, Gatt MK, Archambault V, Lada K, Ichihara K et al. Drosophila Larp associates with poly $(A)$-binding protein and is required for male fertility and syncytial embryo development. Dev Biol. 2009;334:186-97. 33.

871 Bolger AM, Lohse $\mathrm{M}$ and Usadel B. Trimmomatic: a flexible trimmer for Illumina sequence data. Bioinformatics. 2014;30:2114-20.

873 Breitbart $\mathrm{H}$, Cohen $\mathrm{G}$ and Rubinstein S. Role of actin cytoskeleton in mammalian sperm capacitation and the acrosome reaction. Reproduction. 2005;129:2638. 
876 Capra E, Turri F, Lazzari B, Cremonesi P, Gliozzi TM et al. Small RNA sequencing of cryopreserved semen from single bull revealed altered miRNAs and piRNAs expression between High- and Low-motile sperm populations. Bmc Genomics. 2017;18:14.

880 Carreau S, Bourguiba S, Lambard S, Galeraud-Denis I, Genissel C et al. 881 Reproductive system: aromatase and estrogens. Mol Cell Endocrinol. 882 2002;193:137-43.

883 Cingolani P, Platts A, Wang le L, Coon M, Nguyen T et al. A program for annotating 884 and predicting the effects of single nucleotide polymorphisms, SnpEff: SNPs in the genome of Drosophila melanogaster strain w1118; iso-2; iso-3. Fly

Cho DY, Kim YA and Przytycka TM. Chapter 5: Network Biology Approach to Complex Diseases. PLoS Comput Biol. 2012;8:e1002820.

892 Diniz DB, Lopes MS, Broekhuijse ML, Lopes PS, Harlizius B et al. A genome-wide association study reveals a novel candidate gene for sperm motility in pigs. Anim Reprod Sci. 2014;151:201-7.

895 Dowdle JA, Mehta M, Kass EM, Vuong BQ, Inagaki A et al. Mouse BAZ1A (ACF1) Is 896 Dispensable for Double-Strand Break Repair but Is Essential for Averting 897 Improper Gene Expression during Spermatogenesis. PLoS Genet. 2013;9:e1003945. 
899 Dunleavy JEM, Okuda H, O'Connor AE, Merriner DJ, O'Donnell L et al. Katanin-like

900

901

902

903

904

905

906

907

908

909

910

911

912

913

914

915

916

917

918

919

920

921

2 (KATNAL2) functions in multiple aspects of haploid male germ cell development in the mouse. PLoS Genet. 2017;13:e1007078.

Fang P, Xu W, Li D, Zhao X, Dai J et al. A novel acrosomal protein, IQCF1, involved in sperm capacitation and the acrosome reaction. Andrology. 2015;3:332-44.

Fortes MR, Reverter A, Zhang Y, Collis E, Nagaraj SH et al. Association weight matrix for the genetic dissection of puberty in beef cattle. Proc Natl Acad Sci U S A. 2010;107:13642-7.

Gadea J. Sperm factors related to in vitro and in vivo porcine fertility. Theriogenology. 2005;63:431-44.

Galili T. dendextend: an R package for visualizing, adjusting and comparing trees of hierarchical clustering. Bioinformatics. 2015;31:3718-20.

Gao N, Chen Y, Liu X, Zhao Y, Zhu L et al. Weighted single-step GWAS identified candidate genes associated with semen traits in a Duroc boar population. Bmc Genomics. 2019;20:797.

Gòdia M, Estill M, Castelló A, Balasch S, Rodríguez-Gil JE et al. A RNA-Seq Analysis to Describe the Boar Sperm Transcriptome and Its Seasonal Changes. Front Genet. 2019a;10:299.

Gòdia M, Castelló A, Rocco M, Cabrera B, Rodríguez-Gil JE et al. Identification of circular RNAs in porcine sperm and their relation to sperm motility. bioRxiv. $2019 b: 608026$.

Gòdia M, Swanson G and Krawetz SA. A history of why fathers' RNA matters. Biol Reprod. 2018a;99:147-59. 
922 Gòdia M, Mayer FQ, Nafissi J, Castelló A, Rodríguez-Gil JE et al. A technical assessment of the porcine ejaculated spermatozoa for a sperm-specific RNAseq analysis. Syst Biol Reprod Med. 2018b;64:291-303.

Goto M, O'Brien DA and Eddy EM. Speriolin is a novel human and mouse sperm centrosome protein. Hum Reprod. 2010;25:1884-94.

Gunawan A, Kaewmala K, Uddin MJ, Cinar MU, Tesfaye D et al. Association study and expression analysis of porcine ESR1 as a candidate gene for boar fertility and sperm quality. Anim Reprod Sci. 2011;128:11-21.

930 Harper C, Wootton L, Michelangeli F, Lefièvre L, Barratt C et al. Secretory pathway Ca2+-ATPase (SPCA1) Ca2+ pumps, not SERCAs, regulate complex [Ca2+]i signals in human spermatozoa. J Cell Sci. 2005;118:1673-85.

Heid HW, Figge U, Winter S, Kuhn C, Zimbelmann R et al. Novel actin-related proteins Arp-T1 and Arp-T2 as components of the cytoskeletal calyx of the mammalian sperm head. Exp Cell Res. 2002;279:177-87.

936 Hering DM, Olenski K, Rusc A and Kaminski S. Genome-wide association study for semen volume and total number of sperm in Holstein-Friesian bulls. Anim Reprod Sci. 2014;151:126-30.

Hu H, Miao YR, Jia LH, Yu QY, Zhang Q et al. AnimalTFDB 3.0: a comprehensive resource for annotation and prediction of animal transcription factors. Nucleic Acids Res. 2019a;47:D33-D8.

$942 \mathrm{Hu}$, Cheng S, Wang H, Li X, Liu S et al. Distinct roles of two myosins in C. elegans 943 spermatid differentiation. PLoS Biol. 2019b;17:e3000211.

944 Ibba M, and Söll D. Aminoacyl-tRNA Synthesis. Annu Rev Biochem. 2000;69:617945 50. 
946 Izumiyama T, Minoshima S, Yoshida T and Shimizu N. A novel big protein TPRBK possessing 25 units of TPR motif is essential for the progress of mitosis and cytokinesis. Gene. 2012;511:202-17.

949 Jodar M, Sendler E, Moskovtsev SI, Librach CL, Goodrich R et al. Absence of sperm RNA elements correlates with idiopathic male infertility. Sci Transl Med.

951 2015;7:295re6.

952 Kim D, Langmead B and Salzberg SL. HISAT: a fast spliced aligner with low memory 953 requirements. Nat Methods. 2015;12:357-60.

954 Kim J, Kwon JT, Jeong J, Kim J, Hong SH et al. SPATC1L maintains the integrity of 955 the sperm head-tail junction. EMBO Rep. 2018;19.

956 Kozomara A, and Griffiths-Jones S. miRBase: integrating microRNA annotation and 957 deep-sequencing data. Nucleic Acids Res. 2011;39:D152-7.

Krausz C, Escamilla AR and Chianese C. Genetics of male infertility: from research to clinic. Reproduction. 2015;150:R159-74.

960 Li H, Handsaker B, Wysoker A, Fennell T, Ruan J et al. The Sequence 961 Alignment/Map format and SAMtools. Bioinformatics. 2009;25:2078-9.

962 Li X, Chen G and Yang B. Urea transporter physiology studied in knockout mice. 963 Front Physiol. 2012;3:217.

964 Liu H, Li W, Zhang Y, Zhang ZG, Shang XJ et al. IFT25, an intraflagellar transporter 965 protein dispensable for ciliogenesis in somatic cells, is essential for sperm 966 flagella formation. Biol Reprod. 2017;96:993-1006.

967 Liu M, Guan ZL, Shen Q, Lalor P, Fitzgerald U et al. Ulk4 Is Essential for 968 Ciliogenesis and CSF Flow. J Neurosci. 2016;36:7589-600. 
969 Liu T, Huang $\mathrm{Y}$, Liu J, Zhao $\mathrm{Y}$, Jiang $\mathrm{L}$ et al. MicroRNA-122 influences the

970

971

972

973

974

975

976

977

978

979

980

981

982

983

984

985

986

987

988

989

990

991

992 development of sperm abnormalities from human induced pluripotent stem cells by regulating TNP2 expression. Stem Cells Dev. 2013;22:1839-50.

Liu T, Cheng W, Gao Y, Wang H and Liu Z. Microarray analysis of microRNA expression patterns in the semen of infertile men with semen abnormalities. Mol Med Rep. 2012;6:535-42.

Loges NT, Olbrich H, Fenske L, Mussaffi $\mathrm{H}$, Horvath $\mathrm{J}$ et al. DNAl2 mutations cause primary ciliary dyskinesia with defects in the outer dynein arm. Am J Hum Genet. 2008;83:547-58.

Luangpraseuth-Prosper A, Lesueur E, Jouneau L, Pailhoux E, Cotinot C et al. TOPAZ1, a germ cell specific factor, is essential for male meiotic progression. Dev Biol. 2015;406:158-71.

Marques DBD, Bastiaansen JWM, Broekhuijse M, Lopes MS, Knol EF et al. Weighted single-step GWAS and gene network analysis reveal new candidate genes for semen traits in pigs. Genet Select Evol. 2018;50:40.

Martin M. Cutadapt removes adapter sequences from high-throughput sequencing reads. EMBnet J. 2011;17:10-2.

Martin PM, Carnaud M, del Cano GG, Irondelle M, Irinopoulou $T$ et al. Schwannomin-interacting protein-1 isoform IQCJ-SCHIP-1 is a late component of nodes of Ranvier and axon initial segments. J Neurosci. 2008;28:6111-7.

Mengerink KJ, and Vacquier VD. An ATP-binding cassette transporter is a major glycoprotein of sea urchin sperm membranes. J Biol Chem. 2002;277:4072934. 
993 Morales CR, Marat AL, Ni X, Yu Y, Oko R et al. ATP-binding cassette transporters ABCA1, ABCA7, and ABCG1 in mouse spermatozoa. Biochem Bioph Res Co. 2008;376:472-7.

Moraru A, Cakan-Akdogan G, Strassburger K, Males M, Mueller S et al. THADA Regulates the Organismal Balance between Energy Storage and Heat Production. Dev Cell. 2017;41:72-81.

Nagarajan P, Onami TM, Rajagopalan S, Kania S, Donnell R et al. Role of chromodomain helicase DNA-binding protein 2 in DNA damage response signaling and tumorigenesis. Oncogene. 2009;28:1053-62.

1002 Olson GE, Winfrey VP and Nagdas SK. Structural modification of the hamster sperm acrosome during posttesticular development in the epididymis. Microsc Res Tech. 2003;61:46-55.

Pertea M, Pertea GM, Antonescu CM, Chang TC, Mendell JT et al. StringTie enables improved reconstruction of a transcriptome from RNA-seq reads. Nat Biotechnol. 2015;33:290-5.

1008 Publicover S, Harper CV and Barratt C. [Ca2+]i signalling in sperm - making the most of what you've got. Nature Cell Biology. 2007;9:235-42.

1010 Purcell S, Neale B, Todd-Brown K, Thomas L, Ferreira MA et al. PLINK: a tool set for whole-genome association and population-based linkage analyses. Am $\mathrm{J}$ Hum Genet. 2007;81:559-75.

1013 Qin Y, Ji J, Du G, Wu W, Dai J et al. Comprehensive pathway-based analysis identifies associations of BCL2, GNAO1 and CHD2 with non-obstructive azoospermia risk. Hum Reprod. 2014;29:860-6. 
1016 Quintero-Moreno A, Rigau T and Rodriguez-Gil JE. Regression analyses and motile sperm subpopulation structure study as improving tools in boar semen quality analysis. Theriogenology. 2004;61:673-90.

1019 R Developmental Core Team. R: A language and environment for statistical $1020 \quad$ computing. 2010.

1021 Racki LR, Yang JG, Naber N, Partensky PD, Acevedo A et al. The chromatin remodeller ACF acts as a dimeric motor to space nucleosomes. Nature. 2009;462:1016-21.

1024 Ramayo-Caldas Y, Renand G, Ballester M, Saintilan R and Rocha D. Multi-breed and multi-trait co-association analysis of meat tenderness and other meat quality traits in three French beef cattle breeds. Genet Select Evol. 2016;48:37.

Ramayo-Caldas Y, Marmol-Sanchez E, Ballester M, Sanchez JP, Gonzalez-Prendes $\mathrm{R}$ et al. Integrating genome-wide co-association and gene expression to identify putative regulators and predictors of feed efficiency in pigs. Genet Sel Evol. 2019;51:48.

Reverter A, and Fortes MR. Association weight matrix: a network-based approach towards functional genome-wide association studies. Methods Mol Biol.

1035 Reverter A, Barris W, McWilliam S, Byrne KA, Wang $\mathrm{YH}$ et al. Validation of alternative methods of data normalization in gene co-expression studies. Bioinformatics. 2005;21:1112-20.

Reverter A, and Chan EK. Combining partial correlation and an information theory approach to the reversed engineering of gene co-expression networks. Bioinformatics. 2008;24:2491-7. 
1041 Robinson JA, and Buhr MM. Impact of genetic selection on management of boar replacement. Theriogenology. 2005;63:668-78.

1043 Rueda A, Barturen G, Lebron R, Gomez-Martin C, Alganza A et al. sRNAtoolbox: an integrated collection of small RNA research tools. Nucleic Acids Res. 2015;43:W467-73.

1047

Salas-Huetos A, Blanco J, Vidal F, Godo A, Grossmann M et al. Spermatozoa from patients with seminal alterations exhibit a differential micro-ribonucleic acid profile. Fertil Steril. 2015;104:591-601.

Shannon P, Markiel A, Ozier O, Baliga NS, Wang JT et al. Cytoscape: a software environment for integrated models of biomolecular interaction networks. Genome Res. 2003;13:2498-504.

Simon P, Baumner S, Busch O, Rohrich R, Kaese M et al. Polysialic Acid Is Present in Mammalian Semen as a Post-translational Modification of the Neural Cell Adhesion Molecule NCAM and the Polysialyltransferase ST8Siall. J Biol Chem. 2013;288:18825-33.

Smital J, Wolf $\mathrm{J}$ and De Sousa LL. Estimation of genetic parameters of semen characteristics and reproductive traits in Al boars. Anim Reprod Sci. 2005;86:119-30.

1059

Snelling WM, Cushman RA, Keele JW, Maltecca C, Thomas MG et al. Breeding and Genetics Symposium: networks and pathways to guide genomic selection. J Anim Sci. 2013;91:537-52.

1062

Song Z. Roles of the nucleotide sugar transporters (SLC35 family) in health and disease. Mol Aspects Med. 2013;34:590-600.

1064 Suh KS, Tatunchak TT, Crutchley JM, Edwards LE, Marin KG et al. Genomic structure and promoter analysis of PKC-delta. Genomics. 2003;82:57-67. 
1066 Sun XH, Zhu YY, Wang L, Liu HL, Ling Y et al. The Catsper channel and its roles in male fertility: a systematic review. Reprod Biol Endocrinol. 2017;15:65.

1068 Suzuki T, Inoue I, Yamagata T, Morita N, Furuichi T et al. Sequential expression of Efhc1/myoclonin1 in choroid plexus and ependymal cell cilia. Biochem Bioph Res Co. 2008;367:226-33.

Suzuki T, Miyamoto H, Nakahari T, Inoue I, Suemoto T et al. Efhc1 deficiency causes spontaneous myoclonus and increased seizure susceptibility. Hum Mol Genet. 2009;18:1099-109.

1074 Taiyun W, and Viliam S, 2017 R package "corrplot": Visualization of a Correlation Matrix (Version 0.84). Available at: https://github.com/taiyun/corrplot

Tao X, West AE, Chen WG, Corfas G and Greenberg ME. A calcium-responsive transcription factor, CaRF, that regulates neuronal activity-dependent expression of BDNF. Neuron. 2002;33:383-95.

1079 Tian H, Li ZL, Peng D, Bai XG and Liang WB. Expression difference of miR-10b and miR-135b between the fertile and infertile semen samples (p). Forens Sci IntGen S. 2017;6:E257-E9.

Turner SD. qqman: an $R$ package for visualizing GWAS results using $Q-Q$ and manhattan plots. bioRxiv. 2014:005165.

Visconti PE, Westbrook VA, Chertihin O, Demarco I, Sleight S et al. Novel signaling

Vitavska O, and Wieczorek $\mathrm{H}$. Putative role of an SLC45 $\mathrm{H}(+)$ /sugar cotransporter in mammalian spermatozoa. Pflug Arch Eur J Phy. 2017;469:1433-42. 
1089 Waldron A, Wilcox C, Francklyn C and Ebert A. Knock-Down of Histidyl-tRNA Synthetase Causes Cell Cycle Arrest and Apoptosis of Neuronal Progenitor Cells in vivo. Front Cell Dev Biol. 2019;7:67.

1092

1093

1094

1095

1096

1097

1098

1099

1100

1101

1102

1103

1104

1105

1106

1107

1108

1109

1110

1111

Wang X, Yang C, Guo F, Zhang Y, Ju Z et al. Integrated analysis of mRNAs and long noncoding RNAs in the semen from Holstein bulls with high and low sperm motility. Sci Rep. 2019;9:2092.

Westlake CJ, Baye LM, Nachury MV, Wright KJ, Ervin KE et al. Primary cilia membrane assembly is initiated by Rab11 and transport protein particle II (TRAPPII) complex-dependent trafficking of Rabin8 to the centrosome. Proc Natl Acad Sci U S A. 2011;108:2759-64.

Wolf J. Genetic Parameters for Semen Traits in Al Boars Estimated from Data on Individual Ejaculates. Reprod Domest Anim. 2009;44:338-44.

Wu FJ, Lin TY, Sung LY, Chang WF, Wu PC et al. BMP8A sustains spermatogenesis by activating both SMAD1/5/8 and SMAD2/3 in spermatogonia. Sci Signal. 2017;10:eaal1910.

Yang J, Lee SH, Goddard ME and Visscher PM. GCTA: a tool for genome-wide complex trait analysis. Am J Hum Genet. 2011;88:76-82.

Zhao X, Zhao K, Ren J, Zhang F, Jiang C et al. An imputation-based genome-wide association study on traits related to male reproduction in a White Duroc $\mathrm{x}$ Erhualian F2 population. Anim Sci J. 2016;87:646-54.

Zhao Y, Gao N, Li X, El-Ashram S, Wang Z et al. Identifying candidate genes associated with sperm morphology abnormalities using weighted single-step GWAS in a Duroc boar population. Theriogenology. 2020;141:9-15. 
1112 Zheng H, Zhou X, Li DK, Yang F, Pan H et al. Genome-wide alteration in DNA 1113 hydroxymethylation in the sperm from bisphenol A-exposed men. PLoS One. 2017;12:e0178535.

1115 Zhou JH, Zhou QZ, Yang JK, Lyu XM, Bian J et al. MicroRNA-27a-mediated 1116 repression of cysteine-rich secretory protein 2 translation in 1117 asthenoteratozoospermic patients. Asian J Androl. 2017;19:591-5.

1119 Figure legends:

1120 Figure 1 (TIFF)

1121 Manhattan plots depicting the genetic associations between SNPs and the sperm 1122 quality traits that showed genome-wide significant values. Significant associations 1123 have been found with the percentage of: A) Percentage of cells with head 1124 abnormalities (HABN); B) Percentage of cells with abnormal acrosomes after 5 min 1125 incubation at $37^{\circ} \mathrm{C}\left(\mathrm{ACRO} \_5\right)$; C) Percentage of cells with of neck abnormalities 1126 (NABN); D) Percentage of motile spermatozoa after 5 min incubation at $37^{\circ} \mathrm{C}$ 1127 (MT_5); E) Percentage of motile spermatozoa after 90 min incubation at $37^{\circ} \mathrm{C}$ 1128 (MT_90); F) Percentage of cells with proximal droplets (PDROP); G) Ratio of the 1129 percentage of abnormal acrosomes at $5 \mathrm{~min}$ versus $90 \mathrm{~min}$ incubation times 1130 (R_ACRO). The $x$-axis represents chromosome length (Mb), and the $y$-axis shows 1131 the negative $\log _{10} \mathrm{P}$-values of the genetic associations. The horizontal red line 1132 represents the significance threshold (FDR $\leq 0.05)$.

1133

1134 Figure 2 (TIFF)

1135 Co-association network based on the AWM and trancriptomics data. A) Full network 1136 with 1,313 genes and 94 miRNAs. B) Subset of the network showing the 
1137 transcription factor $C A R F$ and all its predicted interactions. C) Subset of the network

1138 with the TRAPPC2L interactions, which included several miRNAs. D) Subset of the

1139 network with the CHD2 gene interactions. The node color corresponds to the

1140 phenotype group with the highest correlation value, as follows: concentration (red),

1141 abnormal acrosomes (green), abnormalities and droplets (pink), osmotic resistance

1142 test (orange), motility (light blue) and viability (dark blue). miRNAs are depicted in

1143 yellow. Node size and text correspond to the number of significant phenotypes

1144 correlated with that gene or miRNA. Nodes with a black line border correspond to

1145 genes identified in the shared network. Node shape indicates classification as:

1146 triangle (TF), V (TF co-factor) and ellipse (other genes and miRNAs).

1147

1148 Additional files:

1149 Additional figure 1 (TIFF)

1150 Outline of the analysis pipeline.

1151 Framework of the dataset, analyses and methodologies included in the study.

1152 Additional figure 2 (PNG)

1153 Correlation across boar sperm quality traits.

1154 Heatmap plot of the correlations among the 25 sperm traits measured on 300 boars.

$1155 \mathrm{CON}=$ Concentration; VIAB_5 = Viability $5 \mathrm{~min}$; VIAB_90= Viability $90 \mathrm{~min} ; \mathrm{ORT}=$

1156 Osmotic Resistance Test; HABN= Head abnormalities; NABN= Neck abnormalities;

$1157 \mathrm{TABN}=$ Tail abnormalities; $\mathrm{PDROP}=$ Proximal droplets; $\mathrm{DDROP}=$ Distal droplets;

1158 MT_5= Motility $5 \mathrm{~min}$; VAP_5= Average Path Velocity $5 \mathrm{~min}$; VCL_5= Curvilinear

1159 Velocity 5 min; $5=$ Straight Line Velocity 5 min; MT_90= Motility 90 min; VAP_90=

1160 Average Path Velocity 90 min; VCL_90= Curvilinear Velocity 90 min; VSL_90= 
1161 Straight Line Velocity 90 min; ACRO_5= Abnormal Abnormal Acrosomes 5 min;

1162 ACRO_90= Abnormal Acrosomes $90 \mathrm{~min} ; \mathrm{R} \_\mathrm{MT}=$ Ratio Motility; R_VAP= Ratio

1163 Average Path Velocity; R_VCL= Ratio Curvilinear Velocity; R_VSL= Ratio Straight

1164 Line Velocity; R_VIAB= Ratio Viability; R_ACRO= Ratio Acrosomes.

1165 Additional figure 3 (PNG)

1166 SNP based dendrogram for the 25 semen parameters.

1167 Dendrogram of the standardized SNP effects across the 25 sperm traits.

1168 Additional file 1 (XLS)

1169 Effect of external factors on sperm quality traits. Effect of farm, age and season per

1170 year across the sperm quality related phenotypes. ${ }^{*}=\mathrm{P}$-value $<0.05 ;{ }^{* *}=\mathrm{P}$-value $<$ $11710.001 ;{ }^{* * *}=$ P-value $<0.0001 ;$ ns=Not Significant.

1172 Additional file 2 (XLS)

1173 Details on the SNPs showing significant associations (FDR $\leq 0.05$ ) in the GWAS

1174 across autosomal chromosomes and unplaced scaffolds. Chr: chromosome; BP:

1175 base pairs (location); Beta = additive effect; FDR = False Discovery Rate; HABN =

1176 Head abnormalities; MT_5 = Percentage of motile spermatozoa at 5 min; MT_90 =

1177 Percentage of motile spermatozoa at $90 \mathrm{~min}$; NABN= Neck abnormalities; PDROP=

1178 Proximal droplets; $R \_A C R O=$ Ratio Abnormal Acrosomes.

1179 Additional file 3 (XLS)

1180 Details of the RNA-seq extraction and mapping statistics. Average and Standard

1181 Deviation (SD) for the 40 samples processed, including the amount of RNA obtained

1182 and several bioinformatics statistics for total RNA-seq (40 samples) and short RNA-

1183 seq (34 samples) datasets. 
1184 Additional file 4 (XLS)

1185 List of protein coding genes and miRNAs identified in sperm. Average and Standard

1186 Deviation (SD) for the samples processed. Protein coding and miRNA abundances

1187 are expressed in Fragments Per Kilobase per Million mapped reads (FPKM) and

1188 counts per million (CPM), respectively.

1189 Additional file 5 (XLS)

1190 SNPs identified in the RNA-seq data mapping within the GWAS regions.

1191 Chr=chromosome. LD=linkage disequilibrium. Allelic frequency for each of the

1192 genotypes. \# samples called=number of samples with reads in the given SNP

1193 position.

1194 Additional file 6 (XLS)

1195 Correlations between gene abundances and phenotypes. P-values are given when

1196 (P-value $\leq$ 0.05). The correlation value is indicated between brackets. ns=Not

1197 Significant.

1198 Additional file 7 (XLS)

1199 Correlations between miRNA abundances and phenotypes. P-values are given when 1200 (P-value < 0.05). The correlation value is indicated between brackets. ns=Not 1201 Significant.

1202 Additional file 8 (XLS)

1203 Associations identified in the within trait eGWAS. Thirty-nine SNPs showed 1204 significant associations (FDR $\leq 0.05)$ with semen phenotypes in the GWAS and also 1205 displayed significant association with the abundance of genes which abundance 1206 correlated with the same phenotype (P-value $\leq 0.05)$. Chr: chromosome. FDR = 
1207 False Discovery Rate; ACRO_5 = Abnormal Acrosomes 5 min; HABN = Head 1208 abnormalities.

1209 Additional file 9 (XLSX)

1210 Gene Ontology analysis of the genes included in the Final Network. GO biological

1211 process terms with significant Bonferroni corrected P-values and their associated 1212 genes.

1213 Additional file 10 (PDF)

1214 Parameter estimates for the significant RNA models. For each of the phenotypes, 1215 the model outputs the estimated values for the 10 genes obtained from the GRM 1216 regression analysis. The lower the value of $\operatorname{Pr}>|t|$, the higher the involvement of the 1217 gene abundance on the total phenotypic variance.

1218 Additional file 11 (XLS)

1219 Description of the SNPs included in the SNP panel. Chromosome, position, SNP ID 1220 and analysis from which the SNP was extracted. 
Table 1. Descriptive statistics, genomic heritability (h2) and number of significant SNPs in the GWAS for sperm quality parameters $(n=300)$.

\begin{tabular}{|c|c|c|c|c|c|}
\hline Trait & Acronym & Mean (SD) & $h_{2}$ (SE) & $\begin{array}{l}\text { Number of SNPs in } \\
\text { autosomal } \\
\text { chromosomes }\end{array}$ & $\begin{array}{l}\text { Number of SNPs } \\
\text { in unplaced } \\
\text { scaffolds }\end{array}$ \\
\hline Concentration (sperm/ml) & CON & $141.3(65.5)$ & $0.13(0.11)$ & 0 & 0 \\
\hline Viability 5 min & VIAB_5 & $90.1(6.3)$ & $1 \times 10-6(0.11)$ & 0 & 0 \\
\hline Viability $90 \mathrm{~min}$ & VIAB_90 & $77.4(17.3)$ & $0.14(0.13)$ & 0 & 0 \\
\hline Osmotic Resistance Test & ORT & $79.8(12.5)$ & $0.13(0.12)$ & 0 & 0 \\
\hline Head abnormalities & HABN & $2.1(5.9)$ & $0.16(0.11)$ & 41 & 0 \\
\hline Neck abnormalities & NABN & $3.0(4.9)$ & $1 \times 10-6(0.13)$ & 18 & 0 \\
\hline Tail abnormalities & TABN & $2.7(3.4)$ & $0.09(0.12)$ & 0 & 0 \\
\hline Proximal droplets & PDROP & $3.5(5.1)$ & $0.12(0.15)$ & 1 & 0 \\
\hline
\end{tabular}




\begin{tabular}{|c|c|c|c|c|c|}
\hline Distal droplets & DDROP & $4.5(4.5)$ & $0.06(0.11)$ & 0 & 0 \\
\hline Motility 5 min & MT_5 & $75.4(18.1)$ & $0.21(0.15)$ & 3 & 217 \\
\hline Motility 90 min & MT_90 & $64.1(22.0)$ & $0.39(0.14)$ & 2 & 252 \\
\hline Average Path Velocity $5 \mathrm{~min}(\mu \mathrm{m} / \mathrm{seg})$ & VAP_5 & $34.0(10.2)$ & $0.17(0.11)$ & 0 & 0 \\
\hline Average Path Velocity $90 \mathrm{~min}(\mu \mathrm{m} / \mathrm{seg})$ & VAP_90 & $30.8(9.5)$ & $0.35(0.13)$ & 0 & 0 \\
\hline Curvilinear Velocity $5 \min (\mu \mathrm{m} / \mathrm{seg})$ & VCL_5 & $46.2(12.5)$ & $0.11(0.10)$ & 0 & 0 \\
\hline Curvilinear Velocity 90 min $(\mu \mathrm{m} / \mathrm{seg})$ & VCL_90 & $39.7(10.2)$ & $0.35(0.13)$ & 0 & 0 \\
\hline Straight Line Velocity 5 min ( $\mu \mathrm{m} / \mathrm{seg})$ & VSL_5 & $27.0(8.3)$ & $0.23(0.13)$ & 0 & 38 \\
\hline Straight Line Velocity $90 \mathrm{~min}(\mu \mathrm{m} / \mathrm{seg})$ & VSL_90 & $25.9(8.3)$ & $0.34(0.13)$ & 0 & 0 \\
\hline Abnormal Acrosomes 5 min & ACRO_5 & $7.0(5.6)$ & $0.08(0.11)$ & 4 & 0 \\
\hline Abnormal Acrosomes 90 min & ACRO_90 & $16.4(12.6)$ & $0.06(0.10)$ & 0 & 0 \\
\hline Ratio Motility & R_MT & $0.9(0.2)$ & $1 \times 10-6(0.11)$ & 0 & 0 \\
\hline
\end{tabular}




\begin{tabular}{lccccc} 
Ratio Average Path Velocity & R_VAP & $0.9(0.3)$ & $1 \times 10-6(0.08)$ & 0 \\
Ratio Curvilinear Velocity & R_VCL & $0.9(0.3)$ & $1 \times 10-6(0.09)$ & 0 & 0 \\
Ratio Straight Line Velocity & R_VSL & $1.0(0.3)$ & $0.06(0.10)$ & 0 & 0 \\
Ratio Viability & R_VIAB & $0.9(0.3)$ & $0.08(0.11)$ & 0 \\
Ratio Acrosomes & R_ACRO & $3.4(3.5)$ & $0.08(0.11)$ & 1 \\
\hline
\end{tabular}

All traits except stated are presented as a percentage. \# SNPs = GWAS number of single nucleotide polymorphisms significantly associated (FDR) with the trait; The values shown are raw excepting the ratios which were previously corrected and stabilized. SD: Standard Deviation; SE: Standard Error 
Table 2. Summary of the results of the genome wide association analysis for sperm quality traits.

\begin{tabular}{|c|c|c|c|c|c|c|c|c|c|c|}
\hline SSC & Interval & \#SNP & Interval, Mbp & Top SNP & $\begin{array}{l}\text { Top SNP } \\
\text { location, bp }\end{array}$ & $\begin{array}{l}\text { Top SNP } \\
\text { P-value }\end{array}$ & $\begin{array}{l}\text { Top SNP } \\
\text { FDR }\end{array}$ & $\begin{array}{l}\text { Top } \\
\text { SNP } \\
\text { MAF }\end{array}$ & Beta & Trait \\
\hline 1 & 11 & 1 & - & rs339761632 & $13,501,755$ & $4.64 \times 10-8$ & 0.02 & 0.06 & 4.84 & PDROP \\
\hline 1 & 12 & 8 & $82.90-83.49$ & rs81354986 & $82,895,619$ & $1.69 \times 10-6$ & 0.03 & 0.07 & 5.05 & HABN \\
\hline 1 & 13 & 8 & $94.88-98.74$ & rs327733412 & $94,880,167$ & $1.61 \times 10-7$ & 0.02 & 0.07 & 5.65 & HABN \\
\hline 1 & 14 & 1 & - & rs337166779 & $126,397,198$ & $2.05 \times 10-6$ & 0.03 & 0.06 & 5.02 & HABN \\
\hline 1 & 15 & 11 & $243.86-246.44$ & rs343194423 & $246,224,386$ & $1.72 \times 10-7$ & 0.01 & 0.07 & 3.17 & NABN \\
\hline 1 & 16 & 2 & 258.54-258.55 & rs332256425 & $258,548,786$ & $1.76 \times 10-6$ & 0.04 & 0.06 & 3.44 & NABN \\
\hline 3 & 11 & 1 & - & rs332055717 & $2,911,413$ & $6.35 \times 10-8$ & 0.01 & 0.09 & 5.07 & HABN \\
\hline 3 & 12 & 3 & $113.75-113.84$ & rs328292697 & $113,750,595$ & $1.09 \times 10-7$ & 0.01 & 0.07 & 3.41 & NABN \\
\hline
\end{tabular}




\begin{tabular}{|c|c|c|c|c|c|c|c|c|c|c|}
\hline 4 & 11 & 2 & $2.41-2.42$ & $\begin{array}{l}\text { rs318575212, } \\
\text { rs332927981 }\end{array}$ & $\begin{array}{l}2,412,006 \\
2,415,239\end{array}$ & $2.88 \times 10-8$ & 0.01 & 0.08 & 4.11 & ACRO_5 \\
\hline 6 & 11 & 2 & $65.60-66.66$ & rs335394654 & $65,597,553$ & $1.86 \times 10-7$ & 0.03 & 0.14 & 3.04 & ACRO_5 \\
\hline 7 & 11 & 2 & $6.20-6.38$ & rs326239534 & $6,377,172$ & $9.87 \times 10-6$ & 0.02 & 0.17 & -9.15 & MT_5 \\
\hline 7 & 12 & 2 & $85.73-86.88$ & rs336588919 & $86,884,279$ & $4.13 \times 10-8$ & 0.01 & 0.06 & 3.75 & NABN \\
\hline 9 & 11 & 2 & $5.76-5.78$ & rs1110111787 & $5,776,597$ & $1.55 \times 10-7$ & 0.02 & 0.07 & 5.43 & HABN \\
\hline 9 & 12 & 1 & - & rs342738178 & $28,463,580$ & $1.53 \times 10-5$ & 0.03 & 0.14 & -10.42 & $\begin{array}{l}\text { MT_5, } \\
\text { MT_90 }\end{array}$ \\
\hline 9 & 13 & 1 & - & rs328217450 & $137,959,590$ & $4.77 \times 10-8$ & 0.02 & 0.18 & 2.36 & R_ACRO \\
\hline 13 & 11 & 18 & $25.36-28.47$ & rs690794887 & $25,535,100$ & $3.06 \times 10-7$ & 0.02 & 0.14 & 3.78 & HABN \\
\hline 13 & 12 & 3 & $33.82-37.65$ & rs327865244 & $33,819,549$ & $3.79 \times 10-8$ & 0.01 & 0.15 & 4.28 & HABN \\
\hline 16 & 11 & 1 & - & rs324239602 & $6,476,358$ & $6.08 \times 10-6$ & 0.01 & 0.46 & 9.07 & MT_90 \\
\hline
\end{tabular}


SSC = S. scrofa chromosome; \#SNP = number of single nucleotide polymorphisms significantly associated (FDR) with the trait; Interval = region of the GWAS interval; Beta = additive effect; FDR = False Discovery Rate; MAF = Minor Allele Frequency; ACRO_5 = Abnormal Acrosomes 5 min; HABN = Head abnormalities; NABN = Neck abnormalities; PDROP = Proximal droplets; R_ACRO = Ratio Acrosomes; MT_5 = Motility 5 min; MT_90 = Motility 90 min. 
Table 3. Summary of the SNPs identified from the RNA-seq datasets in genes mapping within the GWAS regions.

\begin{tabular}{|c|c|c|c|c|c|c|c|c|c|c|}
\hline SSC & Interval & $\begin{array}{l}\text { Top SNP of } \\
\text { the GWAS } \\
\text { interval }\end{array}$ & $\begin{array}{l}\text { \# SNPs } \\
\text { called }\end{array}$ & $\begin{array}{l}\text { Highest } \\
\text { LD }\end{array}$ & $\begin{array}{l}\text { SNP with } \\
\text { highest LD }\end{array}$ & $\begin{array}{l}\text { Gentoypic frequency } \\
(0 / 0 ; 0 / 1 ; 1 / 1)\end{array}$ & $\begin{array}{l}\text { \# called } \\
\text { samples }\end{array}$ & $\begin{array}{l}\text { SNP } \\
\text { effect }\end{array}$ & Gene & Trait \\
\hline 1 & 13 & rs327733412 & 3 & 0.07 & rs710447566 & $0.34 ; 0.54 ; 0.11$ & 35 & Low & KATNAL2 & HABN \\
\hline 13 & 11 & rs690794887 & 21 & 0.4 & rs331304027 & $0.06 ; 0.09 ; 0.85$ & 33 & $\begin{array}{l}\text { Moderat } \\
\mathrm{e}\end{array}$ & ULK4 & HABN \\
\hline 13 & 12 & rs327865244 & 11 & 0.2 & rs323872641 & $0.49 ; 0.37 ; 0.14$ & 35 & Low & $A B H D 14 A$ & HABN \\
\hline
\end{tabular}

SSC = S. scrofa chromosome; \#SNP called = number of single nucleotide polymorphisms identified in the SNP calling analysis. LD

= linkage disequilibrium; Allelic frequency for each of the genotypes. \# called samples = number of samples with reads in the given

SNP position. The column SNP effect and gene refer to the SNP with highest LD in the region. HABN = Head abnormalities; NABN

= Neck abnormalities. 
Table 4. Summary of the results from the within-trait expression genome wide association analysis.

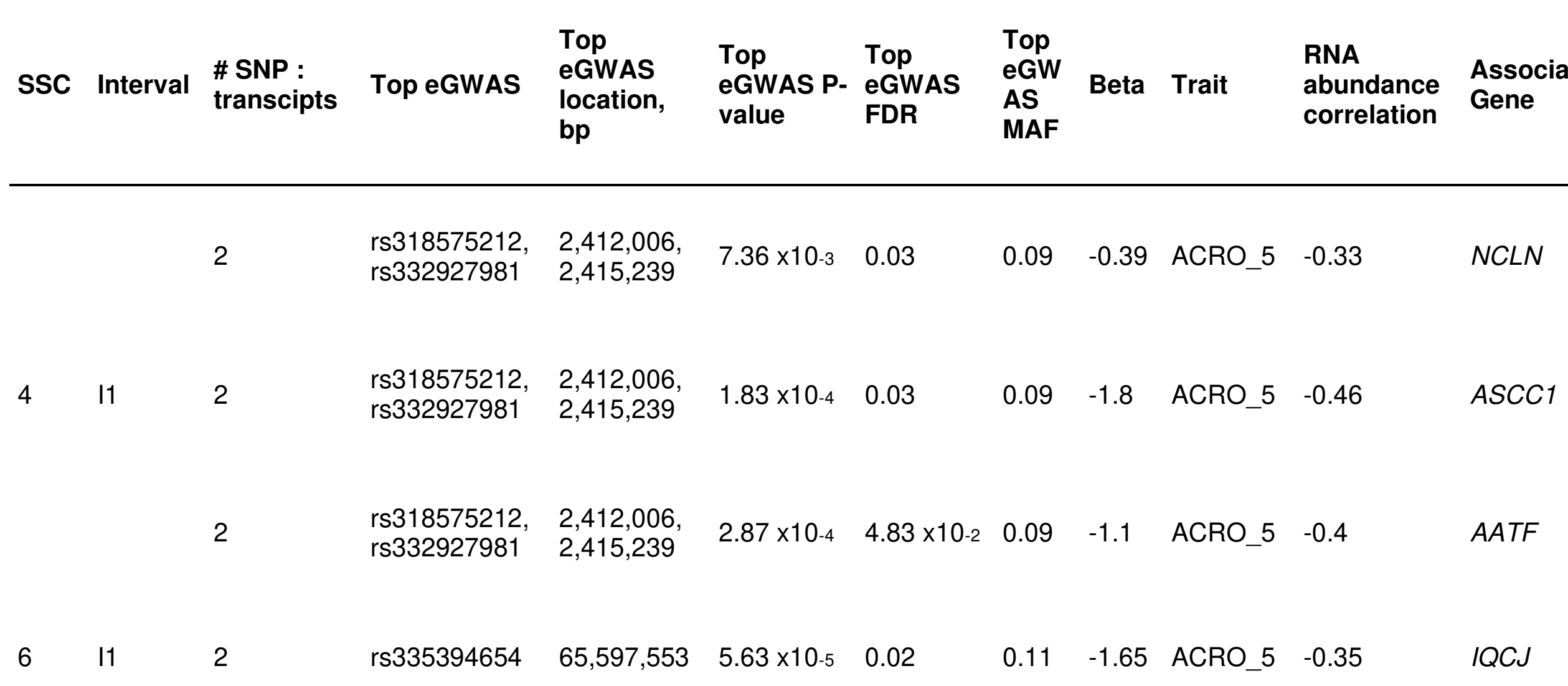


SSC = S. scrofa chromosome; \# SNP : transcripts = number of single nucleotide polymorphisms significantly associated to a transcript; Beta $=$ additive effect; MAF = Minor Allele Frequency; ACRO_5 = Abnormal Acrosomes 5 min; HABN = Head abnormalities. 


\section{Figures}

A)

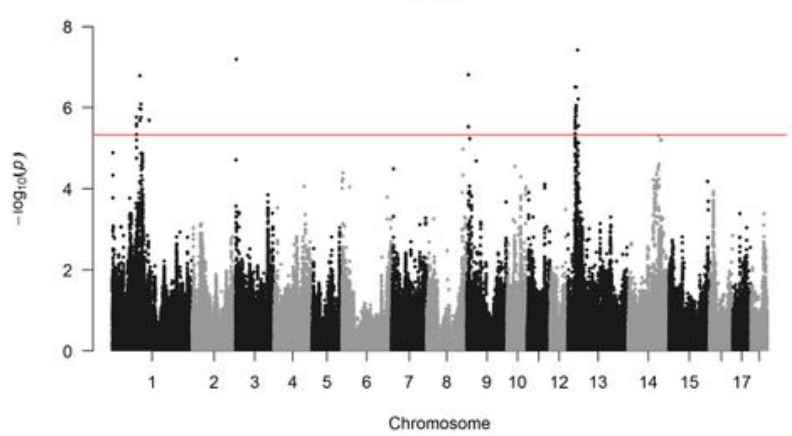

C)

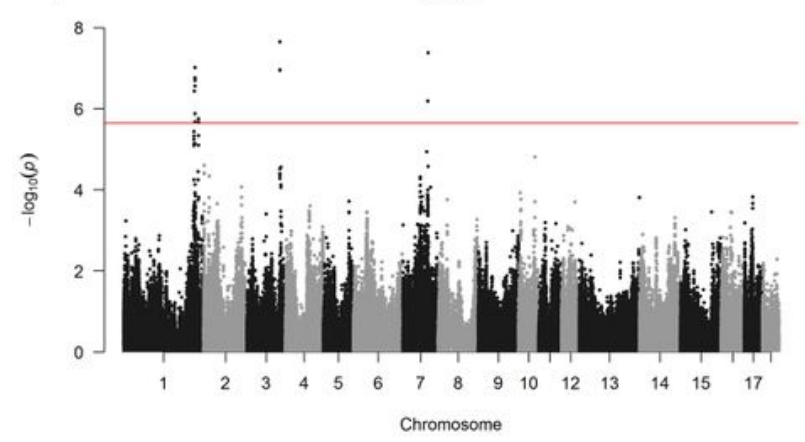

E)

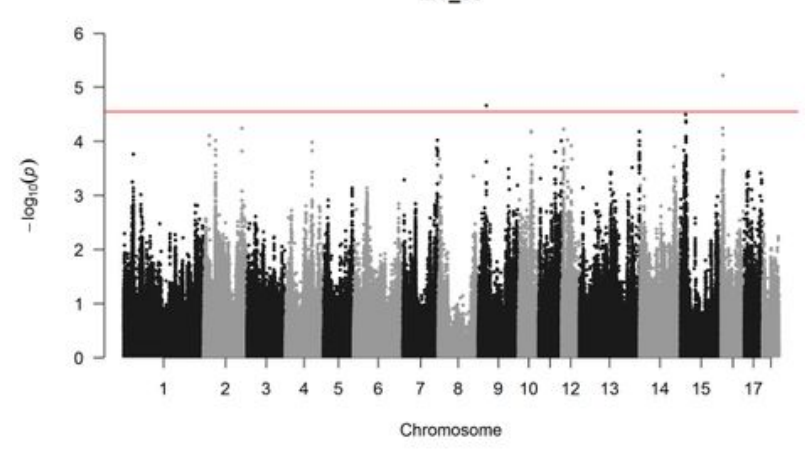

G)
B)

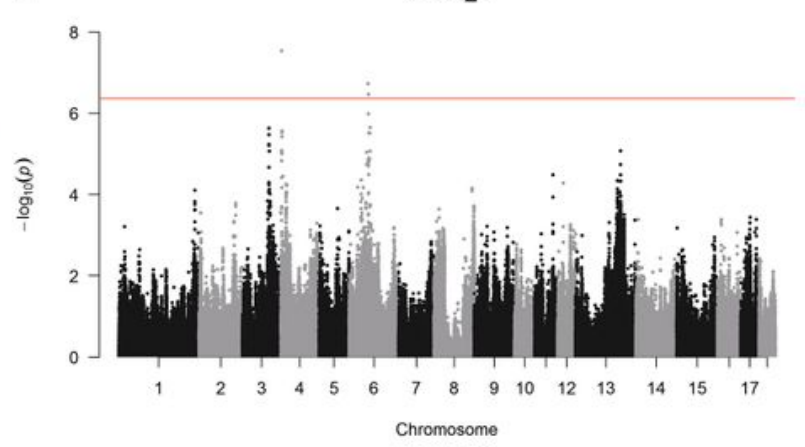

D)
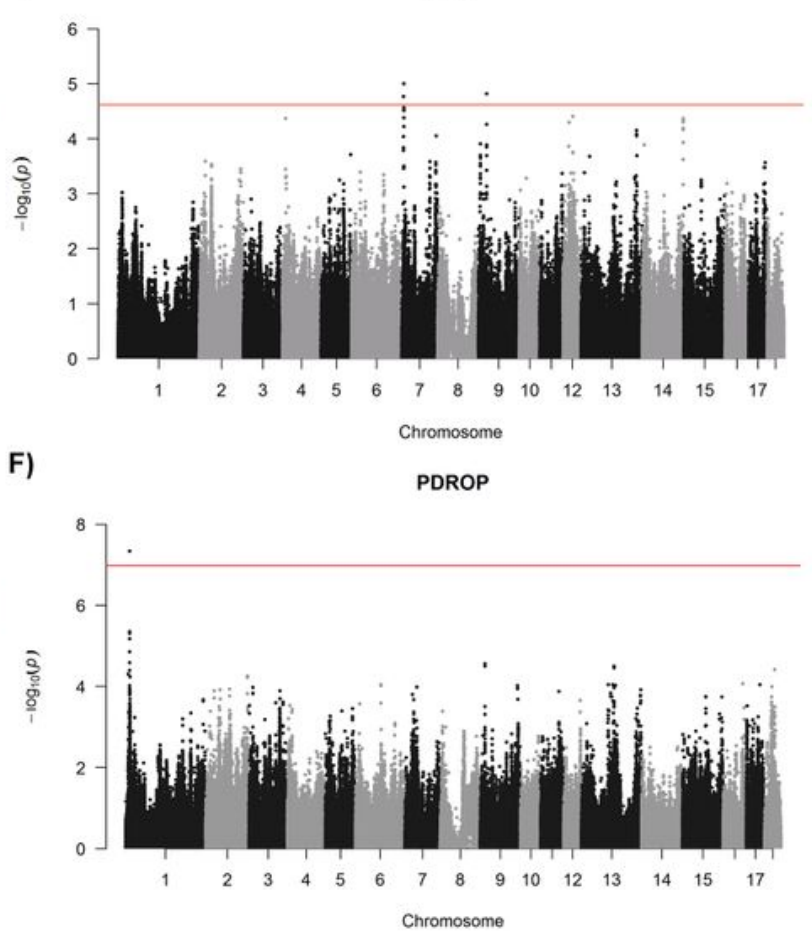

R_ACRO

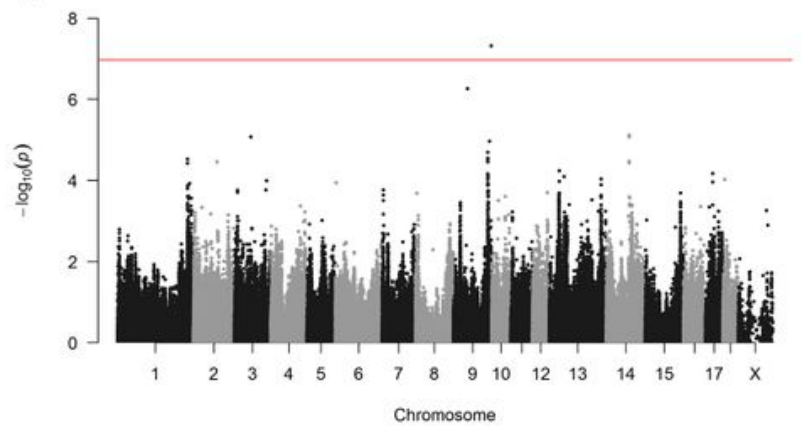

Figure 1

Manhattan plots depicting the genetic associations between SNPs and the sperm quality traits that showed genome-wide significant values. Significant associations have been found with the percentage of: A) Percentage of cells with head abnormalities (HABN); B) Percentage of cells with abnormal 
acrosomes after 5 min incubation at $37^{\circ} \mathrm{C}$ (ACRO_5); C) Percentage of cells with of neck abnormalities (NABN); D) Percentage of motile spermatozoa after 5 min incubation at $37^{\circ} \mathrm{C}$ (MT_5); E) Percentage of motile spermatozoa after 90 min incubation at $37^{\circ} \mathrm{C}$ (MT_90); F) Percentage of cells with proximal droplets (PDROP); G) Ratio of the percentage of abnormal acrosomes at 5 min versus 90 min incubation times (R_ACRO). The $x$-axis represents chromosome length $(\mathrm{Mb})$, and the $y$-axis shows the negative log10 P-values of the genetic associations. The horizontal red line represents the significance threshold (FDR $\leq$ 0.05).

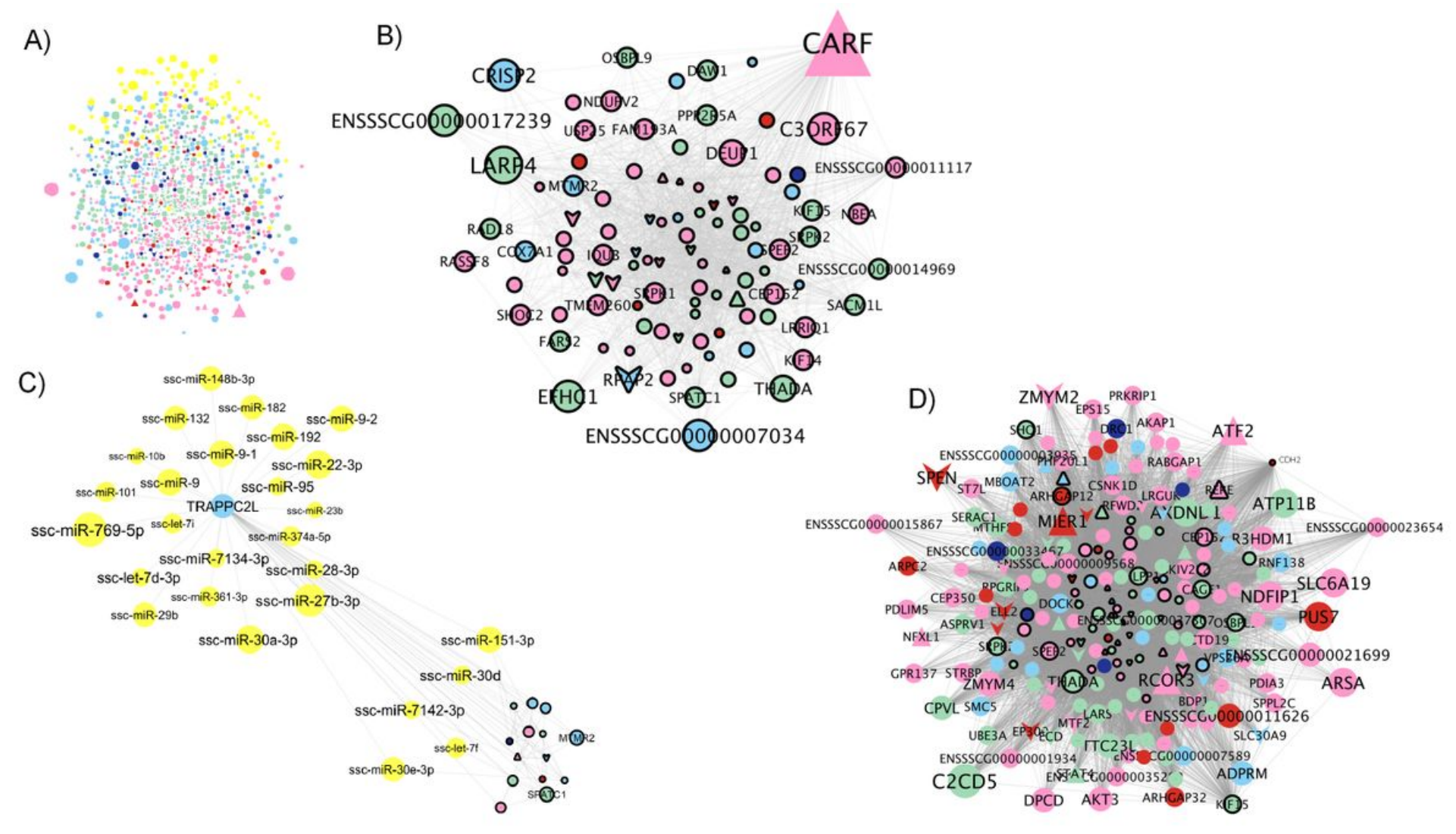

Figure 2

Co-association network based on the AWM and trancriptomics data. A) Full network with 1,313 genes and 94 miRNAs. B) Subset of the network showing the transcription factor CARF and all its predicted interactions. C) Subset of the network with the TRAPPC2L interactions, which included several miRNAs. D) Subset of the network with the CHD2 gene interactions. The node color corresponds to the phenotype group with the highest correlation value, as follows: concentration (red), abnormal acrosomes (green), abnormalities and droplets (pink), osmotic resistance test (orange), motility (light blue) and viability (dark blue). miRNAs are depicted in yellow. Node size and text correspond to the number of significant phenotypes correlated with that gene or miRNA. Nodes with a black line border correspond to genes identified in the shared network. Node shape indicates classification as: triangle (TF), V (TF co-factor) and ellipse (other genes and miRNAs).

\section{Supplementary Files}


This is a list of supplementary files associated with this preprint. Click to download.

- Supplemental.zip 